Pacific

Journal of

Mathematics

\title{
POLES OF CERTAIN RESIDUAL EISENSTEIN SERIES OF CLASSICAL GROUPS
}

\author{
DiHUA JiANG, BAIYING LiU AND LEI ZHANG
}




\title{
POLES OF CERTAIN RESIDUAL EISENSTEIN SERIES OF CLASSICAL GROUPS
}

\author{
DiHUA JiAnG, BAIYING LiU AND LEI ZHANG
}

In memory of Steve Rallis

\begin{abstract}
We study the location of possible poles of a family of residual Eisenstein series on classical groups. Special types of residues of those Eisenstein series were used as key ingredients in the automorphic descent constructions of Ginzburg, Rallis and Soudry and in the refined constructions of Ginzburg, Jiang and Soudry. We study the conditions for the existence of other possible poles of those Eisenstein series and determine the possible Arthur parameters for the residual representations if they exist. Further properties of those residual representations and their applications to automorphic constructions will be considered in our future work.
\end{abstract}

\section{Introduction}

Automorphic descent constructions of Ginzburg, Rallis and Soudry [Ginzburg et al. 2011] produce the inverse of the Langlands functorial transfers from classical groups to the general linear groups. More recently, the extensions of those constructions to produce endoscopy transfers for classical groups were considered in [Ginzburg 2008; Ginzburg et al. 2012; Jiang 2011; 2012]. The key ingredient in these constructions is to use certain Fourier coefficients of special types of residues of certain residual Eisenstein series as kernel functions in the corresponding integral transforms. In order to explore the possibility of more general constructions, in this paper we start to consider other possible poles and residues of these and more general residual Eisenstein series for classical groups.

1A. Classical groups. Let $F$ be a number field and let $E$ be a quadratic extension of $F$ whose Galois group is denoted by $\Gamma_{E / F}=\{1, \iota\}$. Denote by $\mathbb{A}=\mathbb{A}_{F}$ the ring of adeles of $F$.

The classical groups considered in this paper, denoted by $G_{n}$, are the $F$-quasisplit unitary groups $\mathrm{U}_{2 n}$ and $\mathrm{U}_{2 n+1}$ of hermitian type, the $F$-split special orthogonal

The work of Dihua Jiang is supported in part by NSF DMS-1001672.

MSC2010: primary 11F70, 22E50; secondary 22E55, 11F72.

Keywords: residual representations, Arthur parameters, Eisenstein series. 
group $\mathrm{SO}_{2 n+1}$ and the symplectic group $\mathrm{Sp}_{2 n}$, and the $F$-quasisplit even special orthogonal group $\mathrm{SO}_{2 n}$. Define the number field $F^{\prime}$ as $F$ if $G_{n}$ is not a unitary group and as $E$ if it is. Denote by $\mathrm{R}_{F^{\prime} / F}\left(\mathrm{GL}_{n}\right)$ the Weil restriction of the $\mathrm{GL}_{n}$ from $F^{\prime}$ to $F$.

We try to follow closely the notation introduced in [Mœglin and Waldspurger 1995]. Since the groups considered in this paper are quasisplit, we fix a standard Borel subgroup $P_{0}=M_{0} N_{0}$ of $G_{n}$ that is realized in the upper-triangular matrices in a chosen realization of the classical group in matrices [Ginzburg et al. 2011]. Let $T_{0}$ be the maximal split torus of the center of $M_{0}$ that defines the root system $R\left(T_{0}, G_{n}\right)$ with the given positive roots $R^{+}\left(T_{0}, G_{n}\right)$ and the set $\Delta_{0}$ of simple roots. Let $P=M N$ be a standard parabolic subgroup of $G_{n}$ (containing $P_{0}$ ) and let $T_{M}$ be the maximal split torus in the center of $M$. The set of restricted roots is denoted by $R\left(T_{M}, G_{n}\right)$. We define $R^{+}\left(T_{M}, G_{n}\right)$ and $\Delta_{M}$ accordingly.

Furthermore, we define $X_{M}=X_{M}^{G_{n}}$ to be the group of all continuous homomorphisms from $M(\mathbb{A})$ into $\mathbb{C}^{\times}$that are trivial on $M(\mathbb{A})^{1}$. Then following page 6 of [Mœglin and Waldspurger 1995] for the explicit realization of $X_{M}$, define the real part of $X_{M}$, which is denoted by $\operatorname{Re} X_{M}$.

1B. Discrete spectrum of $\mathbf{G L}_{\boldsymbol{a b}}$. Let $\tau$ be an irreducible unitary cuspidal automorphic representation of $\mathrm{GL}_{a}(\mathbb{A})$. Take the standard parabolic subgroup $Q_{a^{b}}=L_{a^{b}} U_{a^{b}}$ of $\mathrm{GL}_{a b}$, whose Levi subgroup $L_{a^{b}}$ is isomorphic to $\mathrm{GL}_{a}^{\times b}$. Then $\pi=\tau^{\otimes b}$ is an irreducible unitary cuspidal automorphic representation of $L_{a^{b}}(\mathbb{A})$. As in Section II.1.5 of [Mœglin and Waldspurger 1995], denote by $\mathfrak{P}$ the $X_{L_{a b}}^{\mathrm{GL}_{a b}}$-orbit of the cuspidal datum $\left(L_{a^{b}}, \pi\right)$. For an automorphic function

$$
\phi_{\pi} \in A\left(L_{a^{b}}(F) U_{a^{b}}(\mathbb{A}) \backslash \mathrm{GL}_{a b}(\mathbb{A})\right)_{\pi},
$$

denote by $\phi_{\pi \otimes \lambda}=\lambda \phi_{\pi}$ the element $\lambda \circ m_{Q} \phi_{\pi}$ for $\lambda \in X_{L_{a} b}^{\mathrm{GL}_{a b}}$. Here the mapping $m_{Q}$ from $\mathrm{GL}_{a b}(\mathbb{A})$ to $L_{a^{b}}(\mathbb{A})^{1} \backslash L_{a^{b}}(\mathbb{A})$ is as defined on page 7 of [Møglin and Waldspurger 1995] by means of the Langlands decomposition with respect to $Q_{a^{b}}(\mathbb{A})$ and the standard maximal compact subgroup of $\mathrm{GL}_{a b}(\mathbb{A})$. An Eisenstein series attached to $\phi_{\pi \otimes \lambda}$ is defined by

$$
E\left(\phi_{\pi \otimes \lambda}, \pi \otimes \lambda\right)(g):=\sum_{\gamma \in Q_{a^{b}}(F) \backslash \mathrm{GL}_{a b}(F)} \lambda \phi_{\pi}(\gamma g) .
$$

It converges absolutely for $\lambda$ in the cone

$$
\left\{\lambda \in \operatorname{Re} X_{L_{a^{b}}}^{\mathrm{GL}_{a b}} \mid\langle\lambda, \tilde{\alpha}\rangle>\left\langle\rho_{Q_{a^{b}}}, \tilde{\alpha}\right\rangle \text { for all } \alpha \in R^{+}\left(T_{L_{a^{b}}}, \mathrm{GL}_{a b}\right)\right\},
$$

and converges uniformly for $g$ in a compact set and $\lambda$ in a neighborhood of 0 in $X_{L_{a b}}^{\mathrm{GL}_{a b}}$. The general theory of Langlands [1976; Mœglin and Waldspurger 1995] asserts that it has meromorphic continuation to the whole parameter space $X_{L_{a} b}^{\mathrm{GL}}$ 
and satisfies the standard functional equations in terms of the relevant intertwining operators.

Take $\Lambda_{b}=((b-1) / 2,(b-3) / 2, \ldots,(1-b) / 2) \in \operatorname{Re} X_{L_{a^{b}}}^{\mathrm{GL}_{a b}}$ and define the iterated residue

$$
\Delta(\tau, b)\left(\phi_{\pi}\right)(g):=\operatorname{Res}_{\Lambda_{b}}^{\mathfrak{P}_{a^{b}}} E\left(\phi_{\pi \otimes \lambda}, \pi \otimes \lambda\right)(g) .
$$

It follows from [Mœglin and Waldspurger 1989] that $\Delta(\tau, b)\left(\phi_{\pi}\right)(g)$ is a squareintegrable automorphic function of $\mathrm{GL}_{a b}(\mathbb{A})$, or more precisely, that it defines the $\mathrm{GL}_{a b}(\mathrm{~A})$-equivariant homomorphism

$$
\Delta(\tau, b): A\left(L_{a^{b}}(F) U_{a^{b}}(\mathbb{A}) \backslash \mathrm{GL}_{a b}(\mathbb{A})\right)_{\pi} \rightarrow L_{\mathrm{disc}}^{2}\left(\mathrm{GL}_{a b}(F) \backslash \mathrm{GL}_{a b}(\mathbb{A})\right)_{\omega_{\tau}^{b}} .
$$

The image is an irreducible subspace of $L_{\text {disc }}^{2}\left(\operatorname{GL}_{a b}(F) \backslash \mathrm{GL}_{a b}(\mathbb{A})\right)_{\omega_{\tau}^{b}}$, which is denoted also by $\mathscr{E}_{(\tau, b)}$, and is usually called the Speh residual representation. Mœglin and Waldspurger proved that all noncuspidal automorphic representations occurring in the discrete spectrum of $\mathrm{GL}_{a b}(\mathbb{A})$ are of this type.

Theorem 1.1 [Mœglin and Waldspurger 1989]. As $b$ ranges over the divisors of $n$, with $n=a b$ and $b>1$, and $\tau$ ranges over the irreducible unitary cuspidal automorphic representations of $\mathrm{GL}_{a}$, with $\omega_{\tau}^{b}=\chi$, the residual representations $\mathscr{E}_{(\tau, b)}$ generated by the corresponding residues $\Delta(\tau, b)\left(\phi_{\pi}\right)$ span the residual spectrum $L_{\mathrm{res}}^{2}\left(\mathrm{GL}_{n}(F) \backslash \mathrm{GL}_{n}(\mathbb{A})\right)_{\chi}$, where $\chi$ is a unitary central character of $\mathrm{GL}_{n}(\mathbb{A})$.

1C. Main results. We consider a family of residual Eisenstein series on $G_{n}(\mathbb{A})$. For a partition $n=r+m$, take the standard maximal parabolic subgroup $P_{r}=M_{r} N_{r}$ of $G_{n}$, whose Levi subgroup $M_{r}$ is isomorphic to $\mathrm{R}_{F^{\prime} / F}\left(\mathrm{GL}_{r}\right) \times G_{m}$. For any $g \in \mathrm{R}_{F^{\prime} / F}\left(\mathrm{GL}_{r}\right)$, define $\hat{g}=w_{r} g^{t} w_{r}$ or $w_{r} \iota(g)^{t} w_{r}$ in the case of unitary groups, where $w_{r}$ is the antidiagonal symmetric matrix defined inductively by

$$
\left(\begin{array}{cc}
0 & 1 \\
w_{r-1} & 0
\end{array}\right)
$$

and $\iota \in \Gamma_{E / F}=\{1, \iota\}$. Then each element $g \in M_{r}$ is of type $\operatorname{diag}\left\{t, h, \hat{t}^{-1}\right\}$, with $t \in \mathrm{R}_{F^{\prime} / F}\left(\mathrm{GL}_{r}\right)$ and $h \in G_{m}$. Since $P_{r}$ is maximal, the space of characters $X_{M_{r}}^{G_{n}}$ is one-dimensional. Using the normalization in [Shahidi 2010], it is identified with $\mathbb{C}$ by $s \mapsto \lambda_{s}$.

For simplicity, we state here only our results for the case of $m>0$, and refer to Section 5 for the case of $m=0$.

Let $\sigma$ be an irreducible generic cuspidal automorphic representation of $G_{m}(\mathbb{A})$. Write $r=a b$. Let $\phi \in A\left(N_{a b}(\mathbb{A}) M_{a b}(F) \backslash G_{n}(\mathbb{A})\right)_{\Delta(\tau, b) \otimes \sigma}$. Following [Langlands 1976; Mœglin and Waldspurger 1995], an Eisenstein series is defined by

$$
E_{a b}^{n}\left(\phi_{\Delta(\tau, b) \otimes \sigma}, s\right)=E\left(\phi_{\Delta \otimes \sigma}, s\right)=\sum_{\gamma \in P_{a b}(F) \backslash G_{n}(F)} \lambda_{s} \phi(\gamma g) .
$$


It converges absolutely for the real part of $s$ large and has meromorphic continuation to the whole complex plane $\mathbb{C}$.

The objective of this paper is to determine the location of possible poles (at $\operatorname{Re}(s) \geq 0)$ of this family of residual Eisenstein series, or more precisely the normalized Eisenstein series, and basic properties of the corresponding residual representations. We take the expected normalizing factor $\beta_{b, \tau, \sigma}(s)$ of the LanglandsShahidi type, which is given by a product of relevant automorphic $L$-functions:

$$
\beta_{b, \tau, \sigma}(s):=L\left(s+\frac{b+1}{2}, \tau \times \sigma\right) \prod_{i=1}^{\lceil b / 2\rceil} L\left(e_{b, i}(s)+1, \tau, \rho\right) \prod_{i=1}^{\lfloor b / 2\rfloor} L\left(e_{b, i}(s), \tau, \rho^{-}\right),
$$

where $e_{b, i}(s):=2 s+b+1-2 i$, and $\rho$ and $\rho^{-}$are defined as

$$
\begin{gathered}
\rho:= \begin{cases}\text { Asai } & \text { if } G_{n}=\mathrm{U}_{2 n}, \\
\text { Asai } \otimes \delta & \text { if } G_{n}=\mathrm{U}_{2 n+1}, \\
\mathrm{Sym}^{2} & \text { if } G_{n}=\mathrm{SO}_{2 n+1}, \\
\Lambda^{2} & \text { if } G_{n}=\mathrm{Sp}_{2 n} \text { or } \mathrm{SO}_{2 n},\end{cases} \\
\rho^{-}:= \begin{cases}\text {Asai } \otimes \delta & \text { if } G_{n}=\mathrm{U}_{2 n}, \\
\text { Asai } & \text { if } G_{n}=\mathrm{U}_{2 n+1}, \\
\Lambda^{2} & \text { if } G_{n}=\mathrm{SO}_{2 n+1}, \\
\operatorname{Sym}^{2} & \text { if } G_{n}=\mathrm{Sp}_{2 n} \text { or } \mathrm{SO}_{2 n} .\end{cases}
\end{gathered}
$$

For unitary groups, "Asai" is the Asai representation of the $L$-group of $R_{E / F}\left(\mathrm{GL}_{a}\right)$ and $\delta$ is the character associated to the quadratic extension $E / F$ via class field theory. For symplectic or orthogonal groups, $\mathrm{Sym}^{2}$ and $\Lambda^{2}$ denote the symmetric and exterior second powers of the standard representation of $\mathrm{GL}_{a}(\mathbb{C})$, respectively. In addition, we have the following identities [Ginzburg et al. 2011, Remark (3), page 21]:

$$
L\left(s, \tau \times \tau^{*}\right)=L(s, \tau, \rho) L\left(s, \tau, \rho^{-}\right),
$$

where $\tau^{*}=\tau$ if $F^{\prime}=F$ and $\tau^{*}=\tau^{\iota}$ if $F^{\prime}=E$, where the involution $\iota$ is the nontrivial element in the Galois group $\Gamma_{E / F}$.

We use the function $\beta_{b, \tau, \sigma}(s)$ to normalize the Eisenstein series by

$$
E_{a b}^{n, *}\left(\phi_{\Delta(\tau, b) \otimes \sigma}, s\right):=\beta_{b, \tau, \sigma}(s) E_{a b}^{n}\left(\phi_{\Delta(\tau, b) \otimes \sigma}, s\right) .
$$

In order to determine the location of the poles of $E^{*}\left(\phi_{\Delta(\tau, b) \otimes \sigma}, s\right)$, we need to consider four cases:

(1) $L(s, \tau, \rho)$ has a pole at $s=1$, and $L\left(\frac{1}{2}, \tau \times \sigma\right) \neq 0$; 
(2) $L(s, \tau, \rho)$ has a pole at $s=1$, and $L\left(\frac{1}{2}, \tau \times \sigma\right)=0$;

(3) $L\left(s, \tau, \rho^{-}\right)$has a pole at $s=1$, and $L(s, \tau \times \sigma)$ has a pole at $s=1$;

(4) $L\left(s, \tau, \rho^{-}\right)$has a pole at $s=1$, and $L(s, \tau \times \sigma)$ is holomorphic at $s=1$.

We define the sets of possible poles according to the four cases:

$$
X_{b, \tau, \sigma}^{+}:= \begin{cases}\left\{\hat{0}, \ldots, \frac{b-2}{2}, \frac{b}{2}\right\} & \text { in Case (1); } \\ \left\{\hat{0}, \ldots, \frac{b-4}{2}, \frac{b-2}{2}\right\} & \text { in Case (2); } \\ \left\{\hat{0}, \ldots, \frac{b-1}{2}, \frac{b+1}{2}\right\} & \text { in Case (3); } \\ \left\{\hat{0}, \ldots, \frac{b-3}{2}, \frac{b-1}{2}\right\} & \text { in Case (4) }\end{cases}
$$

When $b=1$ or 2 , the set $X_{b, \tau, \sigma}^{+}$is empty for Case (2), and when $b=1$, the set $X_{b, \tau, \sigma}^{+}$ is empty for Case (4). Note that we omit 0 in the set $X_{b, \tau, \sigma}^{+}$, since the normalized Eisenstein series $E^{*}\left(\phi_{\Delta(\tau, b) \otimes \sigma}, s\right)$ is holomorphic at $s=0$ (Corollary 4.3).

Theorem 1.2. Assume that $G_{n}$ is either the symplectic group or the $F$-quasisplit special orthogonal group, and assume that $m>0$. Let $\sigma$ be an irreducible generic cuspidal automorphic representation of $G_{m}(\mathbb{A})$, and let $\tau$ be an irreducible unitary self-dual cuspidal automorphic representation of $\mathrm{GL}_{r}(\mathbb{A})$. The normalized Eisenstein series $E_{a b}^{n, *}\left(\phi_{\Delta(\tau, b) \otimes \sigma}, s\right)$ is holomorphic for $\operatorname{Re}(s) \geq 0$ except at $s=s_{0} \in X_{b, \tau, \sigma}^{+}$, where it has possibly at most simple poles.

This is a consequence of Proposition 4.1, Corollary 4.3, and Theorems 4.5 and 5.2.

The proof uses an induction formula (Proposition 3.2) for the constant term of $E^{*}\left(\phi_{\Delta(\tau, b) \otimes \sigma}, s\right)$ along the standard maximal parabolic subgroup $P_{a}$. This formula, which extends a similar one studied in [Jiang 1998], is proved in Section 3, with the unnormalized version proved in Section 2 (Proposition 2.3); it uses the Arthur classification [Arthur 2013] for the discrete spectrum of the classical groups. This yields more explicit information about the residual representations. A special case of $\mathrm{Sp}_{2 n}$ was treated in [Brenner 2009]. We note that there are some mistakes in the arguments used there, and we have corrected them along the way in our discussion.

We remark that the calculations in both Sections 2 and 3 work also for $F$ quasisplit unitary groups, and the results there cover the case when $G_{n}$ is either $\mathrm{U}_{2 n}$ or $\mathrm{U}_{2 n+1}$.

In the proof of Theorem 1.2, the case of $m>0$ is treated in Section 4 and the case of $m=0$ is briefly discussed in Section 5. This makes the discussion clearer and the formulas involved easier to present. By using the induction formula (Proposition 3.2), one reduces the proof to showing that the normalized Eisenstein 
series $E^{*}\left(\phi_{\Delta(\tau, b) \otimes \sigma}, s\right)$ is holomorphic at $0 \leq \operatorname{Re}(s)<\frac{1}{2}$, which is proved in Corollary 4.3 and Proposition 4.4. The proof of this result uses the result of Arthur [2013, Corollary 7.3.5] on behavior at $s=0$ of the normalized intertwining operators, and on classification of the discrete spectrum. We thank James Arthur for his careful explanation of this issue. Since the results in [Arthur 2013] for the case of unitary groups are now proved in [Mok 2012], the proof of Theorem 1.2 also works for $F$-quasisplit unitary groups.

Another issue is to consider the possible poles of the normalized Eisenstein series $E_{a b}^{n, *}\left(\phi_{\Delta(\tau, b) \otimes \sigma}, s\right)$ at $\operatorname{Re}(s)<0$ by the standard functional equation. This needs sufficient properties of the involved standard intertwining operator and the local Plancherel measures in this setting. We will leave this for our future consideration.

There is one more issue in extending Theorem 1.2 to cover the case when $\sigma$ is tempered, but nongeneric. We need to normalize the intertwining operators involved in the calculation of the induction formula so that they are holomorphic and nonzero for $\operatorname{Re}(s)>0$ at every local place. Following the work of Arthur [2013], one is able to define these local $L$-functions at all local places. According to Mœglin [2010], over $p$-adic local fields, for the tempered local $L$-packets, the normalization of these intertwining operators by the Langlands-Shahidi local factors yields the required properties of the normalized intertwining operators. It seems that at archimedean local places, this may need more work, and we decide to consider this technical issue in the future. Hence we still restrict Theorem 1.2 to the generic case in this paper, which is enough for the current applications to our work in progress on constructions of certain types of endoscopy transfers for classical groups [Jiang 2011; 2012].

In Section 4 we prove Theorem 1.2 for the case when $m>0$, and in Section 5 we prove Theorem 1.2 for the case when $m=0$. In the last section, we will discuss the conditions for the existence of poles of the normalized Eisenstein series $E^{*}\left(\phi_{\Delta(\tau, b) \otimes \sigma}, s\right)$ at $s_{0} \in X_{b, \tau, \sigma}^{+}$and determine the possible Arthur parameters for these residual representations of $G_{n}(\mathbb{A})$, which are generated by the residues at $s_{0} \in X_{b, \tau, \sigma}^{+}$, respectively, and are square-integrable.

\section{An induction formula}

In this section, we take $G_{n}$ to be one of the following classical groups: the $F$ quasisplit unitary groups $\mathrm{U}_{2 n}$ and $\mathrm{U}_{2 n+1}$, the $F$-split odd special orthogonal group $\mathrm{SO}_{2 n+1}$, the symplectic group $\mathrm{Sp}_{2 n}$, and the $F$-quasisplit even special orthogonal group $\mathrm{SO}_{2 n}$.

Let $\sigma$ be an irreducible cuspidal automorphic representation of $G_{m}(\mathbb{A})$, without assuming its genericity. From the Langlands theory of Eisenstein series, the possible poles of an Eisenstein series are determined by its constant terms. For the residual 
Eisenstein series $E_{a b}^{n}\left(\phi_{\Delta(\tau, b) \otimes \sigma}, s\right)$, the general formula for constant terms along parabolic subgroups are given in [Mœglin and Waldspurger 1995, Section II.1.7], for instance. In this section, we investigate the constant term of $E_{a b}^{n}\left(\phi_{\Delta(\tau, b) \otimes \sigma}, s\right)$ along the maximal parabolic subgroup $P_{a}$ (as given in Section 1), which leads to an induction formula. This extends the formula in [Jiang 1998] to this more general setting. On the way of our calculations, we also correct some technical mistakes in [Brenner 2009], which treated a special family of residual Eisenstein series of $\mathrm{Sp}_{2 n}(\mathbb{A})$.

In the explicit calculation for the induction formula, we may set $P_{r}^{n}$ for the standard maximal parabolic subgroup $P_{r}$ of $G=G_{n}$. We denote by $Q$ or $Q_{a, a(b-1)}^{a b}$ a parabolic subgroup of $\mathrm{GL}_{a b}$ with Levi subgroup isomorphic to $\mathrm{GL}_{a} \times \mathrm{GL}_{a(b-1)}$.

2A. Constant terms of Eisenstein series. Here we calculate the constant term of $E_{a b}^{n}\left(\phi_{\Delta(\tau, b) \otimes \sigma}, s\right)$ along the maximal parabolic subgroup $P_{a}^{n}=P_{a}$, which is defined by

$$
E_{P_{a}}\left(\phi_{\Delta \otimes \sigma}, s\right)(g)=\int_{N_{a}(F) \backslash N_{a}(\mathbb{A})} E\left(\phi_{\Delta \otimes \sigma}, s\right)(n g) \mathrm{d} n .
$$

Assume that $\operatorname{Re}(s)$ is large. After unfolding the Eisenstein series, we obtain

$$
\begin{aligned}
& E_{P_{a}}\left(\phi_{\Delta \otimes \sigma}, s\right)(g) \\
& \quad=\sum_{w^{-1} \in P_{a b} \backslash G / P_{a} \gamma \in M_{a}^{w}(F) \backslash M_{a}(F)} \int_{\left[N_{a}^{w}\right]} \int_{N_{a, w}(\mathbb{A})} \lambda_{s} \phi\left(w^{-1} \gamma n^{\prime} n^{\prime \prime} g\right) \mathrm{d} n^{\prime} \mathrm{d} n^{\prime \prime},
\end{aligned}
$$

where we define $M_{a}^{w}:=w P_{a b} w^{-1} \cap M_{a}$ and $N_{a}^{w}:=w P_{a b} w^{-1} \cap N_{a}$ and $\left[N_{a}^{w}\right]:=$ $N_{a}^{w}(F) \backslash N_{a}^{w}(\mathbb{A})$. Note that the unipotent radical $N_{a}$ can be decomposed as a product $N_{a, w} N_{a}^{w}$, where $N_{a, w}$ satisfies $N_{a, w} \cap N_{a}^{w}=\{1\}$ and $N_{a}=N_{a, w} N_{a}^{w}=N_{a}^{w} N_{a, w}$.

For the first summation in (2-1), we consider the generalized Bruhat decomposition $P_{a b} \backslash G / P_{a}$. As in [Shahidi 2010, Lemma 4.2.1], the representative $w^{-1}$ of the double coset $P_{a b} w^{-1} P_{a}$ is chosen to have the minimal length. Following the explicit calculations done in [Ginzburg et al. 2011, Chapter 4], it is not hard to figure out that by the cuspidal support of the Eisenstein series, all terms vanish except the two double cosets, whose representatives are given by $w=\mathrm{Id}$ and

$$
w=\omega=(-1)^{a}\left(\begin{array}{ccccc}
0 & 0 & 0 & I_{a} & 0 \\
I_{a(b-1)} & 0 & 0 & 0 & 0 \\
0 & 0 & I & 0 & 0 \\
0 & 0 & 0 & 0 & I_{a(b-1)} \\
0 & \pm I_{a} & 0 & 0 & 0
\end{array}\right),
$$


with $\omega^{-1}$ being in the open cell. Here we use $(-1)^{a}$ and \pm to make sure that $\omega$ belongs to $G_{n}$. Define, for $w=\operatorname{Id}$ or $\omega$,

$$
E_{P_{a}}\left(\phi_{\Delta \otimes \sigma}, s\right)_{w}=\sum_{\gamma \in M_{a}^{w}(F) \backslash M_{a}(F)} \int_{\left[N_{a}^{w}\right]} \int_{N_{a, w}(\mathbb{A})} \lambda_{s} \phi\left(w^{-1} \gamma n^{\prime} n^{\prime \prime} g\right) \mathrm{d} n^{\prime} \mathrm{d} n^{\prime \prime} .
$$

Then the constant term is expressed as

$$
E_{P_{a}}\left(\phi_{\Delta \otimes \sigma}, s\right)=E_{P_{a}}\left(\phi_{\Delta \otimes \sigma}, s\right)_{\mathrm{Id}}+E_{P_{a}}\left(\phi_{\Delta \otimes \sigma}, s\right)_{\omega} .
$$

We will calculate each of these two terms in the following two subsections.

2B. Id-term. Write

$$
n(X, Y, Z, W)=\left(\begin{array}{ccccc}
I_{a} & X & Y & Z & W \\
& I_{a(b-1)} & & & Z^{\prime} \\
& & I & & Y^{\prime} \\
& & & I_{a(b-1)} & X^{\prime} \\
& & & & I_{a}
\end{array}\right) \in N_{a},
$$

where $X^{\prime}, Y^{\prime}$ and $Z^{\prime}$ are uniquely determined by $X, Y$ and $Z$. Note that $P_{a b} \cap$ $M_{a} \backslash M_{a} \cong P_{a(b-1)}^{n-a} \backslash G_{n-a}$. The Id-term of the constant term is

$$
E_{P_{a}}\left(\phi_{\Delta \otimes \sigma}, s\right)_{\mathrm{Id}}(g)=\sum_{\gamma \in P_{a(b-1)}^{n-a}(F) \backslash G_{n-a}(F)} \int_{\left[N_{a}\right]} \lambda_{s} \phi(\gamma n g) \mathrm{d} n,
$$

where $\left[N_{a}\right]:=N_{a}(F) \backslash N_{a}(\mathbb{A})$. The integral can be calculated as follows:

$$
\begin{aligned}
\int_{\left[N_{a}\right]} \lambda_{s} \phi(\gamma n g) \mathrm{d} n & =\int_{\left[N_{a}\right]} \lambda_{s} \phi(n \gamma g) \mathrm{d} n \\
& =\int_{\left[M_{a \times a(b-1)}\right]} \int_{\left[N_{a b} \cap N_{a}\right]} \lambda_{s} \phi\left(n^{\prime} n(X) \gamma g\right) \mathrm{d} n^{\prime} \mathrm{d} X \\
& =\int_{\left[M_{a \times a(b-1)}\right]} \lambda_{s} \phi(n(X) \gamma g) \mathrm{d} X .
\end{aligned}
$$

Here $[Z]:=Z(F) \backslash Z(\mathbb{A})$ for $Z=N_{a}, M_{a \times a(b-1)}$, and $N_{a b} \cap N_{a}$, respectively. We denote by $n(X)$ the element $n(X, 0,0,0)$ with $X \in M_{a \times a(b-1)}$.

Let us understand the last integral

$$
\int_{\left[M_{a \times a(b-1)}\right]} \phi(n(X) g) \mathrm{d} X .
$$

Recall that the Levi subgroup $M_{a b}$ is isomorphic to $\mathrm{R}_{F^{\prime} / F}\left(\mathrm{GL}_{a b}\right) \times G_{m}$. We denote its elements by $(x, h)$ with $x \in \mathrm{R}_{F^{\prime} / F}\left(\mathrm{GL}_{a b}\right)$ and $h \in G_{m}$. We fix $g \in G_{n}(\mathbb{A})$. Then the function

$$
x \mapsto \phi((x, 1) g)
$$


is an automorphic function in the space of the residual representation $\mathscr{E}_{(\tau, b)}$ of $\mathrm{GL}_{a b}\left(\mathbb{A}_{F^{\prime}}\right)$. Consider the standard maximal parabolic subgroup

$$
Q_{a, a(b-1)}=L_{a, a(b-1)} U_{a, a(b-1)}
$$

of $\mathrm{GL}_{a b}$ associated to the partition $a b=a+a(b-1)$. Then the integral (2-4) is the constant term of $\phi((x, 1) g)$ (as an automorphic form in $x$ ) along the maximal parabolic subgroup $Q_{a, a(b-1)}$, which is denoted by $\phi_{Q_{a, a(b-1)}}$.

Let $P_{a, a(b-1)}$ be a standard parabolic subgroup of $G_{n}$ whose Levi subgroup $M_{a, a(b-1)}$ is isomorphic to

$$
\mathrm{R}_{F^{\prime} / F} \mathrm{GL}_{a} \times \mathrm{R}_{F^{\prime} / F} \mathrm{GL}_{a(b-1)} \times G_{m}
$$

and whose unipotent radical is $N:=N_{a, a(b-1)}$. We denote by $(t, r, h)$ the element $\operatorname{diag}\left(t, r, h, \hat{r}^{-1}, \hat{t}^{-1}\right)$ in $M_{a, a(b-1)}(\mathbb{A})$.

Lemma 2.1. The constant term $\lambda_{s} \phi_{Q_{a, a(b-1)}}$ belongs to the space

$$
A\left(N_{a, a(b-1)}(\mathbb{A}) M_{a, a(b-1)}(F) \backslash G_{n}(\mathbb{A})\right)_{\left.\tau|\cdot|\right|_{F^{\prime}} ^{s-(b-1) / 2} \otimes \Delta(\tau, b-1)|\cdot|_{F^{\prime}}^{s+1 / 2} \otimes \sigma} .
$$

Here $|\cdot|_{F^{\prime}}=|\operatorname{det}|_{\mathbb{A}_{F^{\prime}}}$; and $F^{\prime}$ is $E$ if $G_{n}$ is unitary, and is $F$ otherwise.

Proof. Let $\boldsymbol{K}=\Pi_{v} K_{v}$ be the standard choice of maximal compact subgroup of $G_{n}(\mathbb{A})$ such that the Iwasawa decomposition

$$
G_{n}(\mathbb{A})=P_{a, a(b-1)}(\mathbb{A}) K
$$

holds. It suffices to show that for all $k \in \boldsymbol{K}$, the constant term $\lambda_{s} \phi_{Q_{a, a(b-1)}}((t, r, h) k)$ belongs to the space of automorphic forms

$$
A\left(M_{a, a(b-1)}(F) \backslash M_{a, a(b-1)}(\mathbb{A})\right)_{\tau|\cdot| F_{F^{\prime}}^{s-(b-1) / 2} \otimes \Delta(\tau, b-1)|\cdot|_{F^{\prime}}^{s+1 / 2} \otimes \sigma},
$$

where $t \in \mathrm{GL}_{a}\left(\mathbb{A}_{F^{\prime}}\right), r \in \mathrm{GL}_{a(b-1)}\left(\mathbb{A}_{F^{\prime}}\right)$ and $h \in G_{m}(\mathbb{A})$.

By the definition (2-4), we have

$$
\phi_{Q_{a, a(b-1)}}((t, r, h) k)=\int_{\left[M_{a \times a(b-1)}\right]} \phi(n(X)(t, r, h) k) \mathrm{d} X .
$$

Since the function $\phi_{k}(m):=m^{-\rho_{P_{a b}}} \phi(m k)$, for $m \in M_{a b}(\mathbb{A})$, is an automorphic form in $A\left(M_{a b}(F) \backslash M_{a b}(\mathbb{A})\right)_{\Delta(\tau, b) \otimes \sigma}$ for all $k \in \boldsymbol{K}$, without loss of generality, we can assume that

$$
\phi_{k}((t, r, h))=\phi_{k, \Delta(\tau, b)}((t, r)) \otimes \phi_{k, \sigma}(h),
$$

where the function $\phi_{k, \Delta(\tau, b)} \in A\left(\mathrm{GL}_{a b}\left(F^{\prime}\right) \backslash \mathrm{GL}_{a b}\left(\mathbb{A}_{F^{\prime}}\right)\right)_{\Delta(\tau, b)}$ and the function $\phi_{k, \sigma} \in A\left(G_{m}(F) \backslash G_{m}(\mathbb{A})\right)_{\sigma}$. Therefore, we obtain

$$
\phi_{Q_{a, a(b-1)}}((t, r, h) k)=\left(\phi_{k, \Delta(\tau, b)}\right)_{Q_{a, a(b-1)}}((t, r)) \otimes \phi_{k, \sigma}(h),
$$


where $\left(\phi_{k, \Delta(\tau, b)}\right)_{Q_{a, a(b-1)}}$ is the constant term of $\phi_{k, \Delta(\tau, b)}$ along the parabolic subgroup $Q_{a, a(b-1)}$ of $\mathrm{GL}_{a b}$.

By [Jiang and Liu 2012, Lemma 4.1], the constant term $\left(\phi_{k, \Delta(\tau, b)}\right)_{Q_{a, a(b-1)}}$ belongs to the space

$$
A\left(U_{a, a(b-1)}(\mathbb{A}) L_{a, a(b-1)}(F) \backslash \mathrm{GL}_{a b}\left(\mathbb{A}_{F^{\prime}}\right)\right)_{|\cdot|_{F^{\prime}}^{-(b-1) / 2} \tau \otimes|\cdot|_{F^{\prime}}^{1 / 2} \Delta(\tau, b-1)} .
$$

It follows that the function $\lambda_{s} \phi_{Q_{a, a(b-1)}}(g)$ belongs to the space

$$
A\left(N_{a, a(b-1)}(\mathbb{A}) M_{a, a(b-1)}(F) \backslash G_{n}(\mathbb{A})\right)_{\tau|\cdot|_{F^{\prime}}^{s-(b-1) / 2} \otimes \Delta(\tau, b-1)|\cdot|_{F^{\prime}}^{s+1 / 2} \otimes \sigma} .
$$

According to Lemma 2.1, we restrict the Id-term $E_{a b, P_{a}^{n}}^{n}\left(\phi_{\Delta \otimes \sigma}, s\right)_{\text {Id }}$ to the subgroup $I_{a} \times G_{n-a}(\mathbb{A})$ of the Levi subgroup $\mathrm{GL}_{a}\left(\mathbb{A}_{F^{\prime}}\right) \times G_{n-a}^{a}(\mathbb{A})$ and obtain (2-5)

$$
\begin{aligned}
E_{a b, P_{a}^{n}}^{n}\left(\phi_{\Delta \otimes \sigma}, s\right)_{\operatorname{Id}}\left(\left(I_{a}, h\right)\right) & =\sum_{\gamma \in P_{a(b-1)}^{n-a}(F) \backslash G_{n-a}(F)} \lambda_{s} \phi_{Q_{a, a(b-1)}^{a b}}\left(\operatorname{diag}\left(I_{a}, \gamma h, I_{a}\right)\right) \\
& =E_{a(b-1)}^{n-a}\left(\lambda_{-1 / 2}\left(i_{n-a}^{*} \phi_{Q}\right)_{\Delta(\tau, b-1) \otimes \sigma}, s+\frac{1}{2}\right)(h),
\end{aligned}
$$

where $|\cdot|_{F^{\prime}}:=|\cdot|_{\mathbb{A}_{F^{\prime}}}$ and the restriction $i_{n-a}^{*} \phi_{Q}=i_{n-a}^{*} \phi_{Q_{a, a(b-1)}^{a b}}$ to $G_{n-a}(\mathbb{A})$ is an automorphic function in the space

$$
A\left(N_{a(b-1)}^{n-a}(\mathbb{A}) M_{a(b-1)}^{n-a}(F) \backslash G_{n-a}(\mathbb{A})\right)_{\Delta(\tau, b-1)|\cdot|_{F^{\prime}}^{1 / 2} \otimes \sigma} .
$$

2C. $\omega$-term. It is easy to see that

$$
N_{a}^{\omega}=\left\{n(0,0, Z, 0) \mid Z \in M_{a \times a(b-1)}\right\} .
$$

We denote by $\tilde{n}(Z)$ the element $n(0,0, Z, 0)$. The coset $M_{a}^{\omega}(F) \backslash M_{a}(F)$ is isomorphic to $P_{a(b-1)}(F) \backslash G_{n-a}(F)$. Therefore, we have $E_{P_{a}}\left(\phi_{\Delta \otimes \sigma}, s\right)_{\omega}(g)$

$$
\begin{aligned}
& =\sum_{\gamma \in P_{a(b-1)}^{n-a}(F) \backslash G_{n-a}(F)} \int_{N_{a, \omega}(\mathbb{A})} \int_{\left[M_{a \times a(b-1)}\right]} \lambda_{s} \phi\left(\omega^{-1} \gamma \tilde{n}(Z) n g\right) \mathrm{d} Z \mathrm{~d} n \\
& =\sum_{\gamma \in P_{a(b-1)}^{n-a}(F) \backslash G_{n-a}(F)} \int_{N_{a, \omega}(\mathbb{A})} \int_{\left[M_{a(b-1) \times a}\right]} \lambda_{s} \phi\left(n(Z) \omega^{-1} n \gamma g\right) \mathrm{d} Z \mathrm{~d} n,
\end{aligned}
$$

where $\left[M_{a(b-1) \times a}\right]:=M_{a(b-1) \times a}(F) \backslash M_{a(b-1) \times a}(\mathbb{A})$, and $n(Z)$ is the element

$$
\left(\begin{array}{ccccc}
I_{a(b-1)} & Z & & & \\
& I_{a} & & & \\
& & I & & \\
& & I_{a} & Z^{\prime} \\
& & & I_{a(b-1)}
\end{array}\right) \text { for } Z \in M_{a(b-1) \times a} .
$$


We denote the inner integration by

$$
\tilde{\phi}(g):=\int_{\left[M_{a(b-1) \times a]}\right.} \phi(n(Z) g) \mathrm{d} Z .
$$

Let $Q_{a(b-1), a}:=L_{a(b-1), a} U_{a(b-1), a}$ be a standard parabolic subgroup of $\mathrm{GL}_{a b}$ whose unipotent radical $U_{a(b-1), a}$ embedded into $G_{n}$ consists of all the elements $n(Z)$. Moreover, the standard parabolic subgroup $P_{a(b-1), a}=M_{a(b-1), a} N_{a(b-1), a}$ of $G_{n}$ has the property that $M_{a(b-1), a}=L_{a(b-1), a} \times G_{m}$ and $N_{a(b-1), a}=U_{a(b-1), a} N_{a b}$.

Lemma 2.2. The function $\lambda_{s} \tilde{\phi}$ is an automorphic function in the space

$$
A\left(N_{a(b-1), a}(\mathbb{A}) M_{a(b-1), a}(F) \backslash G_{n}(\mathbb{A})\right)_{\left.|\cdot|\right|_{F^{\prime}} ^{s-1 / 2} \Delta(\tau, b-1) \otimes|\cdot|_{F^{\prime}}^{s+(b-1) / 2} \tau \otimes \sigma} .
$$

Here $|\cdot|_{F^{\prime}}$ is as defined in Lemma 2.1.

Proof. The proof is similar to the proof of Lemma 2.1. For all $k \in \boldsymbol{K}$, the function $\phi_{k}(m):=m^{-\rho_{P a b}} \phi(m k)$, for $m \in M_{a b}(\mathbb{A})$, is an automorphic form in the space $A\left(M_{a b}(F) \backslash M_{a b}(\mathbb{A})\right)_{\Delta(\tau, b) \otimes \sigma}$. We may assume that

$$
\phi_{k}((t, r, h))=\phi_{k, \Delta(\tau, b)}((t, r)) \otimes \phi_{k, \sigma}(h),
$$

where $t \in \mathrm{GL}_{a(b-1)}\left(\mathbb{A}_{F^{\prime}}\right), r \in \mathrm{GL}_{a}\left(\mathbb{A}_{F^{\prime}}\right)$ and $h \in G_{m}(\mathbb{A})$. Then

$$
\tilde{\phi}_{k}((t, r, h))=\left[\left(\phi_{k, \Delta(\tau, b)}\right)_{Q_{a(b-1), a}}((t, r)) \otimes \phi_{k, \sigma}(h)\right] .
$$

By [Jiang and Liu 2012, Lemma 4.1], the constant term $\left(\phi_{k, \Delta(\tau, b)}\right)_{Q_{a(b-1), a}}$ is an automorphic function in the space

$$
A\left(U_{a(b-1), a}(\mathbb{A}) L_{a(b-1), a}(F) \backslash \mathrm{GL}_{a b}\left(\mathbb{A}_{F^{\prime}}\right)\right)_{|\cdot| \cdot F^{\prime}}^{-1 / 2} \Delta(\tau, b-1) \otimes|\cdot|_{F^{\prime}}^{(b-1) / 2} \tau .
$$

This is enough to deduce the lemma.

Next, following the notation of [Mœglin and Waldspurger 1995, II.1.6], we consider the intertwining operator

$$
M(\omega, \cdot):=M\left(\omega,|\cdot|_{F^{\prime}}^{s-1 / 2} \Delta(\tau, b-1) \otimes|\cdot|_{F^{\prime}}^{s+(b-1) / 2} \tau \otimes \sigma\right),
$$

which is defined by

$$
\left(M(\omega, \cdot) \lambda_{s} \tilde{\phi}\right)(g):=\int_{N_{a, a(b-1)} \cap \omega N_{a(b-1), a} \omega^{-1}(F) \backslash N_{a, a(b-1)}(\mathbb{A})} \lambda_{s} \tilde{\phi}\left(\omega^{-1} n g\right) \mathrm{d} n .
$$

Now, plug this intertwining operator into the $\omega$-term and obtain

$$
E_{P_{a}}\left(\phi_{\Delta \otimes \sigma}, s\right)_{\omega}(g)=\sum_{\gamma \in P_{a(b-1)}^{n-a}(F) \backslash G_{n-a}(F)}\left(M(\omega, \cdot) \lambda_{s} \tilde{\phi}\right)(\gamma g) .
$$


By [Mœglin and Waldspurger 1995, Proposition II.1.6], the intertwining operator $M(\omega, \cdot)$ maps

$$
A\left(N_{a(b-1), a}(\mathbb{A}) M_{a(b-1), a}(F) \backslash G_{n}(\mathbb{A})\right)_{|\cdot|_{F^{\prime}}^{s-1 / 2} \Delta(\tau, b-1) \otimes|\cdot|_{F^{\prime}}^{s+(b-1) / 2} \tau \otimes \sigma}
$$

to

$$
A\left(N_{a, a(b-1)}(\mathbb{A}) M_{a, a(b-1)}(F) \backslash G_{n}(\mathbb{A})\right)_{|\cdot|_{F^{\prime}}^{-(s+(b-1) / 2)} \tilde{\tau}^{*} \otimes|\cdot|_{F^{\prime}}^{s-1 / 2} \Delta(\tau, b-1) \otimes \sigma},
$$

where $\tau^{*}=\tau$ if $F^{\prime}=F$, and $\tau^{*}=\tau^{\iota}$ if $F^{\prime}=E$, with $\iota$ being the nontrivial element in the Galois group $\Gamma_{E / F}$. Therefore, the restriction of the $\omega$-term $E_{a b, P_{a}}^{n}\left(\phi_{\Delta \otimes \sigma}, s\right)_{\omega}$ to the subgroup $I_{a} \times G_{n-a}(\mathbb{A})$ of the Levi subgroup $\mathrm{GL}_{a}\left(\mathbb{A}_{F^{\prime}}\right) \times G_{n-a}(\mathbb{A})$ is equal to

$$
E_{a(b-1)}^{n-a}\left(\lambda_{1 / 2}\left(i_{n-a}^{*} \circ M(\omega, \cdot)\right) \tilde{\phi}, s-\frac{1}{2}\right)(h) .
$$

Combining the results of Sections $2 \mathrm{~B}$ and $2 \mathrm{C}$, we achieve an induction formula of the constant term.

Proposition 2.3. The constant term $E_{a b, P_{a}}^{n}\left(\phi_{\Delta(\tau, b) \otimes \sigma}, s\right)$ restricted to the subgroup $I_{a} \times G_{n-a}(\mathbb{A})$ of the Levi subgroup $\mathrm{GL}_{a}\left(\mathbb{A}_{F^{\prime}}\right) \times G_{n-a}(\mathbb{A})$ is expressed as the identity

$$
\begin{aligned}
& E_{a b, P_{a}}^{n}\left(\phi_{\Delta \otimes \sigma}, s\right)\left(\left(I_{a}, h\right)\right) \\
& =E_{a(b-1)}^{n-a}\left(\lambda_{-1 / 2}\left(i_{n-a}^{*} \phi_{Q_{a, a(b-1)}^{a b}}\right)_{\Delta(\tau, b-1) \otimes \sigma}, s+\frac{1}{2}\right)(h) \\
& +E_{a(b-1)}^{n-a}\left(\lambda_{1 / 2}\left(i_{n-a}^{*} \circ M(\omega, \cdot)\right) \tilde{\phi}, s-\frac{1}{2}\right)(h),
\end{aligned}
$$

which holds for all $s$ with $\operatorname{Re}(s)$ large, and then is extended to $s \in \mathbb{C}$ by meromorphic continuation. Here

$$
M(\omega, \cdot):=M\left(\omega,|\cdot|_{F^{\prime}}^{s-1 / 2} \Delta(\tau, b-1) \otimes|\cdot|_{F^{\prime}}^{s+(b-1) / 2} \tau \otimes \sigma\right),
$$

$|\cdot|_{F^{\prime}}:=|\cdot|_{\mathbb{A}_{F^{\prime}}}$, and $\tilde{\phi}$ is defined in (2-6). Note that $F^{\prime}$ is $E$ if $G_{n}$ is a unitary group, and is $F$ otherwise.

\section{A normalized induction formula}

In this section, we keep the assumption on $G_{n}$ as in Section 2 and calculate normalizing factors for the relevant intertwining operators involved in the functional equation of Eisenstein series and in the induction formula (2-9). This leads to an induction formula for normalized Eisenstein series. As we remarked in the introduction of this paper, we have to assume that $\sigma$ is an irreducible generic cuspidal automorphic representation of $G_{m}(\mathbb{A})$ if $m>0$. 
3A. Normalized Eisenstein series $(\boldsymbol{m}>\mathbf{0})$. We assume that $m>0$, and recall the definitions of $\rho$ and $\rho^{-}$in (1-2) and (1-3):

$$
\rho:= \begin{cases}\text { Asai } & \text { if } G_{n}=\mathrm{U}_{2 n}, \\ \text { Asai } \otimes \delta & \text { if } G_{n}=\mathrm{U}_{2 n+1}, \\ \mathrm{Sym}^{2} & \text { if } G_{n}=\mathrm{SO}_{2 n+1}, \\ \Lambda^{2} & \text { if } G_{n}=\mathrm{Sp}_{2 n} \text { or } \mathrm{SO}_{2 n},\end{cases}
$$

and

$$
\rho^{-}:= \begin{cases}\text {Asai } \otimes \delta & \text { if } G_{n}=\mathrm{U}_{2 n}, \\ \text { Asai } & \text { if } G_{n}=\mathrm{U}_{2 n+1}, \\ \Lambda^{2} & \text { if } G_{n}=\mathrm{SO}_{2 n+1}, \\ \operatorname{Sym}^{2} & \text { if } G_{n}=\mathrm{Sp}_{2 n} \text { or } \mathrm{SO}_{2 n} .\end{cases}
$$

It follows [Ginzburg et al. 2011, Remark (3)] that

$$
L\left(s, \tau \times \tau^{*}\right)=L(s, \tau, \rho) L\left(s, \tau, \rho^{-}\right),
$$

where $\tau^{*}=\tau$ if $F^{\prime}=F$ and $\tau^{*}=\tau^{\iota}$ if $F^{\prime}=E$, where the involution $\iota$ is the nontrivial element in the Galois group $\Gamma_{E / F}$.

In order to normalize the Eisenstein series, we consider the normalization of the intertwining operator $M\left(\omega^{\prime},|\cdot|_{F^{\prime}}^{s} \Delta \otimes \sigma\right)(\phi)$ with

$$
\omega^{\prime}=(-1)^{a b}\left(\begin{array}{ll}
I_{a b} & I \\
\pm I_{a b} &
\end{array}\right) .
$$

By the general theory of Eisenstein series and intertwining operators [Langlands 1976; Mœglin and Waldspurger 1995, Chapter VI; Shahidi 2010, Theorem 6.1.7], both $E\left(\phi_{\Delta(\tau, b) \otimes \sigma}, s\right)$ and $M\left(\omega^{\prime},|\cdot|_{F^{\prime}}^{s} \Delta \otimes \sigma\right)$ can be extended to meromorphic functions of $s \in \mathbb{C}$, and the Eisenstein series $E\left(\phi_{\Delta \otimes \sigma}, s\right)$ has the functional equation

$$
E\left(\phi_{\Delta(\tau, b) \otimes \sigma}, s\right)=E\left(M\left(\omega^{\prime},|\cdot|_{F^{\prime}}^{s} \Delta(\tau, b) \otimes \sigma\right)(\phi),-s\right) .
$$

If $\operatorname{Re}(s)=0$, then $E\left(\phi_{\Delta(\tau, b) \otimes \sigma}, s\right)$ is holomorphic.

For any factorizable function $\phi=\bigotimes_{v} \phi_{v}$, we write

$$
M\left(\omega^{\prime},|\cdot|_{F^{\prime}}^{s} \Delta \otimes \sigma\right)(\phi)=\prod_{v} M\left(\omega^{\prime},|\cdot|_{F_{v}^{\prime}}^{s} \Delta_{v} \otimes \sigma_{v}\right)\left(\phi_{v}\right) .
$$

By [Shahidi 2010, Theorem 6.3.1], for each local place $v$, define

$$
\begin{aligned}
N^{\prime}\left(\omega^{\prime},|\cdot|_{F_{v}^{\prime}}^{s} \Delta\left(\tau_{v}, b\right)\right. & \left.\otimes \sigma_{v}\right)\left(\phi_{v}\right) \\
= & \frac{1}{r^{\prime}\left(\omega^{\prime},|\cdot|_{F_{v}^{\prime}}^{s} \Delta\left(\tau_{v}, b\right) \otimes \sigma_{v}\right)} M\left(\omega^{\prime},|\cdot|_{F_{v}^{\prime}}^{s} \Delta\left(\tau_{v}, b\right) \otimes \sigma_{v}\right)\left(\phi_{v}\right),
\end{aligned}
$$


where the local normalizing factor $r^{\prime}\left(\omega^{\prime},|\cdot|_{F_{v}^{\prime}}^{s} \Delta\left(\tau_{v}, b\right) \otimes \sigma_{v}\right)$

$$
\begin{aligned}
=\frac{L\left(s, \Delta\left(\tau_{v}, b\right) \times \sigma_{v}\right)}{L\left(s+1, \Delta\left(\tau_{v}, b\right) \times \sigma_{v}\right) \varepsilon\left(s, \Delta\left(\tau_{v}, b\right) \times \sigma_{v}, \psi_{v}\right)} \\
\times \frac{L\left(2 s, \Delta\left(\tau_{v}, b\right), \rho\right)}{L\left(2 s+1, \Delta\left(\tau_{v}, b\right), \rho\right) \varepsilon\left(2 s, \Delta\left(\tau_{v}, b\right), \rho, \psi_{v}\right)} .
\end{aligned}
$$

Define $r^{\prime}\left(\omega^{\prime},|\cdot|_{F^{\prime}}^{s} \Delta(\tau, b) \otimes \sigma\right)=\prod_{v} r^{\prime}\left(\omega^{\prime},|\cdot|_{F_{v}^{\prime}}^{s} \Delta\left(\tau_{v}, b\right) \otimes \sigma_{v}\right)$. The global normalized intertwining operator is

$$
N^{\prime}\left(\omega^{\prime},|\cdot|_{F^{\prime}}^{s} \Delta \otimes \sigma\right)=\prod_{v} N^{\prime}\left(\omega^{\prime},|\cdot|_{F_{v}^{\prime}}^{s} \Delta\left(\tau_{v}, b\right) \otimes \sigma_{v}\right) .
$$

For the global (complete) $L$-functions, we have

$$
\begin{aligned}
L(s, \Delta(\tau, b) \times \sigma)= & \prod_{i=1}^{b} L\left(s+\frac{2 i-b-1}{2}, \tau \times \sigma\right), \\
L(s, \Delta(\tau, b), \rho)= & \prod_{i=1}^{b} L(s+b-2 i+1, \tau, \rho) \\
& \times \prod_{1 \leq i<j \leq b} L\left(s+b-(i+j)+1, \tau \otimes \tau^{*}\right) .
\end{aligned}
$$

Hence the quotient of complete $L$-functions has the property that

$$
\frac{L(s, \Delta(\tau, b) \times \sigma) L(2 s, \Delta(\tau, b), \rho)}{L(s+1, \Delta(\tau, b) \times \sigma) L(2 s+1, \Delta(\tau, b), \rho)}
$$

is equal to

$$
\frac{\prod_{i=1}^{\lceil b / 2\rceil} L\left(f_{b, i}(s), \tau, \rho\right) \prod_{i=1}^{\lfloor b / 2\rfloor} L\left(f_{b, i}(s)+1, \tau, \rho^{-}\right) L\left(s-\frac{b-1}{2}, \tau \times \sigma\right)}{\prod_{i=1}^{\lceil b / 2\rceil} L\left(e_{b, i}(s)+1, \tau, \rho\right) \prod_{i=1}^{\lfloor b / 2\rfloor} L\left(e_{b, i}(s), \tau, \rho^{-}\right) L\left(s+\frac{b+1}{2}, \tau \times \sigma\right)},
$$

where $e_{b, i}(s):=2 s+b+1-2 i, f_{b, i}(s):=2 s-b-1+2 i$. Define

$$
\begin{aligned}
\alpha_{b}(s) & :=\prod_{i=1}^{\lceil b / 2\rceil} L\left(f_{b, i}(s), \tau, \rho\right) \prod_{i=1}^{\lfloor b / 2\rfloor} L\left(f_{b, i}(s)+1, \tau, \rho^{-}\right) L\left(s-\frac{b-1}{2}, \tau \times \sigma\right), \\
\beta_{b}(s) & :=\prod_{i=1}^{\lceil b / 2\rceil} L\left(e_{b, i}(s)+1, \tau, \rho\right) \prod_{i=1}^{\lfloor b / 2\rfloor} L\left(e_{b, i}(s), \tau, \rho^{-}\right) L\left(s+\frac{b+1}{2}, \tau \times \sigma\right),
\end{aligned}
$$


and

$$
\varepsilon_{b}(s):=\prod_{i=1}^{\lceil b / 2\rceil} \varepsilon\left(f_{b, i}(s), \tau, \rho\right) \prod_{i=1}^{\lfloor b / 2\rfloor} \varepsilon\left(f_{b, i}(s)+1, \tau, \rho^{-}\right) \varepsilon\left(s-\frac{b-1}{2}, \tau \times \sigma\right) .
$$

Finally define the global normalizing factor by

$$
r\left(\omega^{\prime},|\cdot|_{F^{\prime}}^{s} \Delta \otimes \sigma\right)=r\left(\omega^{\prime},|\cdot|_{F^{\prime}}^{s} \Delta(\tau, b) \otimes \sigma\right)=\frac{\alpha_{b}(s)}{\beta_{b}(s) \varepsilon_{b}(s)}
$$

and the normalized global intertwining operator by

$$
N\left(\omega^{\prime},|\cdot|_{F^{\prime}}^{s} \Delta \otimes \sigma\right)=\frac{\varepsilon_{b}(s) N^{\prime}\left(\omega^{\prime},|\cdot|_{F^{\prime}}^{s} \Delta \otimes \sigma\right)}{\varepsilon(s, \Delta(\tau, b) \times \sigma) \varepsilon(2 s, \Delta(\tau, b), \rho)} .
$$

Then we have that

$$
N\left(\omega^{\prime},|\cdot|_{F^{\prime}}^{s} \Delta(\tau, b) \otimes \sigma\right)=\frac{1}{r\left(\omega^{\prime},|\cdot|_{F^{\prime}}^{s} \Delta \otimes \sigma\right)} M\left(\omega^{\prime},|\cdot|_{F^{\prime}}^{s} \Delta \otimes \sigma\right) .
$$

Meanwhile, we use $\beta_{b}(s)$ to normalize the Eisenstein series

$$
E_{a b}^{n, *}\left(\phi_{\Delta(\tau, b) \otimes \sigma}, s\right):=\beta_{b}(s) E_{a b}^{n}\left(\phi_{\Delta(\tau, b) \otimes \sigma}, s\right) .
$$

By the functional equation (3-1) of $E_{a b}^{n}\left(\phi_{\Delta(\tau, b) \otimes \sigma}, s\right)$, the normalized Eisenstein series $E_{a b}^{n, *}\left(\phi_{\Delta(\tau, b) \otimes \sigma}, s\right)$ satisfies the functional equation

$$
E_{a b}^{n, *}\left(\phi_{\Delta(\tau, b) \otimes \sigma}, s\right)=E_{a b}^{n, *}\left(N\left(\omega^{\prime},|\cdot|_{F^{\prime}}^{s} \Delta \otimes \sigma\right)(\phi),-s\right) .
$$

In fact,

$$
\begin{aligned}
E^{*}\left(\phi_{\Delta \otimes \sigma}, s\right) & =\beta_{b}(s) E\left(M\left(\omega^{\prime},|\cdot|_{F^{\prime}}^{s} \Delta \otimes \sigma\right)(\phi),-s\right) \\
& =\beta_{b}(s) \cdot r\left(\omega^{\prime},|\cdot|_{F^{\prime}}^{s} \Delta \otimes \sigma\right) E\left(N\left(\omega^{\prime},|\cdot|_{F^{\prime}}^{s} \Delta \otimes \sigma\right)(\phi),-s\right) \\
& =\frac{\beta_{b}(s) \cdot r\left(\omega^{\prime},|\cdot|_{F^{\prime}}^{s} \Delta \otimes \sigma\right)}{\beta_{b}(-s)} E^{*}\left(N\left(\omega^{\prime},|\cdot|_{F^{\prime}}^{s} \Delta \otimes \sigma\right)(\phi),-s\right) .
\end{aligned}
$$

Since $\alpha_{b}(s)=\varepsilon_{b}(s) \beta_{b}(-s)$, we have

$$
\beta_{b}(s) \cdot r\left(\omega^{\prime},|\cdot|_{F^{\prime}}^{s} \Delta \otimes \sigma\right)=\beta_{b}(-s) .
$$

From this we deduce the functional equation (3-6).

We remark that when $b=1$, it is easy to show that for $\operatorname{Re}(s)>0$, the normalized global intertwining operator

$$
N\left(\omega^{\prime},|\cdot|_{F^{\prime}}^{s} \Delta(\tau, b) \otimes \sigma\right)
$$

is holomorphic for all choice of data, and nonzero for some choice of data.

In fact, if $b=1$ and $\tau \otimes \sigma$ is a generic representation, then the normalized local intertwining operator $N\left(\omega^{\prime}, \cdot\right)$ is holomorphic and nonzero by Theorem 11.1 of 
[Cogdell et al. 2004]. The proof uses the Langlands functorial transfers of $\sigma$ from $G_{n}$ to the corresponding general linear groups, the Ramanujan type estimate for cuspidal automorphic forms on general linear groups [Luo et al. 1999], and the structure of generic unitary dual for classical groups over all local fields [Lapid et al. 2004]. Hence the result for $b=1$ holds for $F$-quasisplit unitary groups with the same proof [Cogdell et al. 2011].

However, when $b>1$, we are not able to prove the above properties for the normalized global intertwining operator $N\left(\omega^{\prime}, \cdot\right)$, so that we are not able to control the poles at $\operatorname{Re}(s)<0$ of the normalized Eisenstein series through the functional equation (3-6). We will leave this issue for our future consideration.

3B. Normalization of $M(\omega, \cdot)$ with $m>0$. In order to normalize the global intertwining operator

$$
M(\omega, \cdot):=M\left(\omega,|\cdot|_{F^{\prime}}^{s-1 / 2} \Delta(\tau, b-1) \otimes|\cdot|_{F^{\prime}}^{s+(b-1) / 2} \tau \otimes \sigma\right),
$$

as defined in (2-7), we decompose it into a composition of two intertwining operators

$$
M\left(\omega,|\cdot|_{F^{\prime}}^{s-1 / 2} \Delta(\tau, b-1) \otimes|\cdot|_{F^{\prime}}^{s+(b-1) / 2} \tau \otimes \sigma\right)=M\left(\omega_{1}, \cdot\right) \circ M\left(\omega_{2}, \cdot\right),
$$

where

$$
\omega_{1}=\left(\begin{array}{cccc} 
& I_{a} & & \\
I_{a(b-1)} & & & \\
& & I & \\
& & & I_{a(b-1)} \\
& & I_{a} &
\end{array}\right)
$$

and

$$
\omega_{2}=(-1)^{a}\left(\begin{array}{cccc}
I_{a(b-1)} & & & \\
& & I_{a} & \\
& & I & \\
& \pm I_{a} & & \\
& & & I_{a(b-1)}
\end{array}\right) .
$$

More precisely, $M\left(\omega_{1}, \cdot\right)$ and $M\left(\omega_{2}, \cdot\right)$ are standard intertwining operators of the following types: $M\left(\omega_{2}, \cdot\right)$ maps from the space

$$
A\left(N_{a(b-1), a}(\mathbb{A}) M_{a(b-1), a}(F) \backslash G_{n}(\mathbb{A})\right)_{|\cdot|_{F^{\prime}}^{s-1 / 2} \Delta(\tau, b-1) \otimes|\cdot|_{F^{\prime}}^{s+(b-1) / 2} \tau \otimes \sigma}
$$

to the space

$$
A\left(N_{a(b-1), a}(\mathbb{A}) M_{a(b-1), a}(F) \backslash G_{n}(\mathbb{A})\right)_{|\cdot| F^{\prime}}^{s-1 / 2} \Delta(\tau, b-1) \otimes|\cdot|_{F^{\prime}}^{-s-(b-1) / 2} \tilde{\tau}^{*} \otimes \sigma,
$$

and $M\left(\omega_{1}, \cdot\right)$ maps from the space

$$
A\left(N_{a(b-1), a}(\mathbb{A}) M_{a(b-1), a}(F) \backslash G_{n}(\mathbb{A})\right)_{|\cdot|_{F^{\prime}}^{s-1 / 2} \Delta(\tau, b-1) \otimes|\cdot|_{F^{\prime}}^{-s-(b-1) / 2} \tilde{\tau}^{*} \otimes \sigma}
$$


to the space

$$
A\left(N_{a(b-1), a}(\mathbb{A}) M_{a(b-1), a}(F) \backslash G_{n}(\mathbb{A})\right)_{|\cdot|_{F^{\prime}}^{-s-(b-1) / 2} \tilde{\tau}^{*} \otimes|\cdot|_{F^{\prime}}^{s-1 / 2} \Delta(\tau, b-1) \otimes \sigma} .
$$

The standard Langlands-Shahidi normalizing factors for $M\left(\omega_{1}, \cdot\right)$ and $M\left(\omega_{2}, \cdot\right)$ are given by $r\left(\omega_{1}, \cdot\right)$ and $r\left(\omega_{2}, \cdot\right)$, where

$$
r\left(\omega_{1}, \cdot\right)=\frac{L\left(2 s+\frac{b}{2}-1, \Delta(\tau, b-1) \times \tau^{*}\right)}{L\left(2 s+\frac{b}{2}, \Delta(\tau, b-1) \times \tau^{*}\right) \varepsilon\left(2 s+\frac{b}{2}-1, \Delta(\tau, b-1) \times \tau^{*}\right)}
$$

and $r\left(\omega_{2}, \cdot\right)$ is

$$
\frac{L\left(s+\frac{b-1}{2}, \tau \times \sigma\right) L(2 s+b-1, \tau, \rho)}{L\left(s+\frac{b+1}{2}, \tau \times \sigma\right) L(2 s+b, \tau, \rho) \varepsilon\left(s+\frac{b-1}{2}, \tau \times \sigma\right) \varepsilon(2 s+b-1, \tau, \rho)} .
$$

We define

$$
\begin{gathered}
M\left(\omega_{1}, \cdot\right)=r\left(\omega_{1}, \cdot\right) N\left(\omega_{1}, \cdot\right), \\
M\left(\omega_{2}, \cdot\right)=r\left(\omega_{2}, \cdot\right) N\left(\omega_{2}, \cdot\right), \\
r(\omega, \cdot)=r\left(\omega_{1}, \cdot\right) r\left(\omega_{2}, \cdot\right),
\end{gathered}
$$

and

$$
M(\omega, \cdot)=r(\omega, \cdot) N(\omega, \cdot)
$$

It follows that

$$
N(\omega, \cdot)=N\left(\omega_{1}, \cdot\right) \circ N\left(\omega_{2}, \cdot\right)
$$

Proposition 3.1. Assume that $b>1$. For $\operatorname{Re}(s)>0$, the normalized global intertwining operator

$$
N(\omega, \cdot)=N\left(\omega,|\cdot|_{F^{\prime}}^{s-1 / 2} \Delta(\tau, b-1) \otimes|\cdot|_{F^{\prime}}^{s+(b-1) / 2} \tau \otimes \sigma\right)
$$

is holomorphic for all choices of data, and nonzero for some choice of data. For $\operatorname{Re}(s)=0$, it is holomorphic.

Proof. First we show that the normalized intertwining operators $N\left(\omega_{i}, \cdot\right)$ for $i=1,2$ are holomorphic and nonzero at $\operatorname{Re}(s) \geq 0$.

Indeed, by Theorem 11.1 in [Cogdell et al. 2004] for orthogonal and symplectic group cases, $N\left(\omega_{2}, \cdot\right)$ is holomorphic for all choices of data and nonzero for some choice of data, when $\operatorname{Re}(s+(b-1) / 2) \geq 0$. For even and odd unitary group cases, the same result follows from Proposition 9.4 in [Kim and Krishnamurthy 2005] and Proposition 5 in [Kim and Krishnamurthy 2004]. 
For the normalized intertwining operators $N\left(\omega_{1}, \cdot\right)$, it is essentially the intertwining operator for general linear groups, which is considered in [Mœglin and Waldspurger 1989]. We write it as an eulerian product

$$
N\left(\omega_{1}, \cdot\right)=\prod_{v} N_{v}\left(\omega_{1}, \cdot\right),
$$

with $\tau=\bigotimes_{v} \tau_{v}$. Since $\tau_{v}$ is unitary and generic, we can assume that

$$
\tau_{v}=|\cdot|^{\nu_{1}} \operatorname{St}\left(\tau_{1}, a_{1}\right) \times|\cdot|^{\nu_{2}} \operatorname{St}\left(\tau_{2}, a_{2}\right) \times \cdots \times|\cdot|^{\nu_{r}} \operatorname{St}\left(\tau_{r}, a_{r}\right),
$$

where $-\frac{1}{2}<v_{i}<\frac{1}{2}$ for all $i$ and $\operatorname{St}\left(\tau_{i}, a_{i}\right)$ are Steinberg representations for some supercuspidal representations $\tau_{i}$ and integers $a_{i}$, and nonlinked. Write $e\left(\tau_{v}\right)=$ $2 \inf \left\{\frac{1}{2}-\left|v_{i}\right|, 1 \leq i \leq r\right\}$ (referring to I.10 in [Mœglin and Waldspurger 1989]). It follows that

$$
e\left(\Delta\left(\tau_{v}, b-1\right)\right)=e\left(\tau_{v}\right) .
$$

By Proposition I.10 in [Mœglin and Waldspurger 1989], $N_{v}\left(\omega_{1}, \cdot\right)$ is holomorphic and nonzero when $\operatorname{Re}\left(s-\frac{1}{2}-(-s-(b-1) / 2)\right)>-e\left(\tau_{v}\right)$ at all local places $v$. In particular, they are holomorphic and nonzero at $\operatorname{Re}(s) \geq 0$, and so is the normalized global intertwining operator $N\left(\omega_{1}, \cdot\right)$. Hence $N(\omega, \cdot)=N\left(\omega_{1}, \cdot\right) \circ N\left(\omega_{2}, \cdot\right)$ is holomorphic for all choices of data when $\operatorname{Re}(s) \geq 0$.

We notice that for $\operatorname{Re}(s)>0, N\left(\omega_{1}, \cdot\right)$ as a $\mathrm{GL}_{a b}$-intertwining operator is an isomorphism, and hence $N(\omega, \cdot)=N\left(\omega_{1}, \cdot\right) \circ N\left(\omega_{2}, \cdot\right)$ is nonzero for some choice of data.

By substituting the normalized Eisenstein series $E_{a b}^{n, *}\left(\phi_{\Delta(\tau, b) \otimes \sigma}, s\right)$ in (3-5) and the normalized intertwining operator $N(\omega, \cdot)$ in (3-7) into the induction formula (2-9) in Proposition 2.3, we obtain

$$
\begin{aligned}
& E_{a b, P_{a}}^{n, *}\left(\phi_{\Delta \otimes \sigma}, s\right)\left(\left(I_{a}, h\right)\right) \\
& =\frac{\beta_{b}(s)}{\beta_{b-1}\left(s+\frac{1}{2}\right)} E_{a(b-1)}^{n-a, *}\left(\lambda_{-1 / 2}\left(i_{n-a}^{*} \phi_{Q_{a, a(b-1)}^{a b}}\right)_{\Delta(\tau, b-1) \otimes \sigma}, s+\frac{1}{2}\right)(h) \\
& \quad+\frac{\beta_{b}(s) \cdot r(\omega, \cdot)}{\beta_{b-1}\left(s-\frac{1}{2}\right)} E_{a(b-1)}^{n-a, *}\left(\lambda_{1 / 2}\left(i_{n-a}^{*} \circ N(\omega, \cdot)\right) \tilde{\phi}, s-\frac{1}{2}\right)(h) .
\end{aligned}
$$

Using a similar calculation as in (3-2), it is easy to verify that

$$
\begin{aligned}
\frac{\beta_{b}(s)}{\beta_{b-1}\left(s+\frac{1}{2}\right)} & =L\left(2 s+1, \tau, \rho^{(-)^{b+1}}\right), \\
\frac{\beta_{b}(s)}{\beta_{b-1}\left(s-\frac{1}{2}\right)} r(\omega, \cdot) & =\frac{L\left(2 s, \tau, \rho^{(-)^{b+1}}\right)}{\varepsilon_{b}^{\prime}(s)},
\end{aligned}
$$


where

$$
\varepsilon_{b}^{\prime}(s):=\varepsilon\left(s+\frac{b-1}{2}, \tau \times \sigma\right) \varepsilon(2 s+b-1, \tau, \rho) \varepsilon\left(2 s+\frac{b}{2}-1, \Delta(\tau, b-1) \times \tau^{*}\right) .
$$

Therefore, for $b>1$, we obtain the following normalized induction formula.

Proposition 3.2 (induction formula). Let $G_{n}$ be the classical groups as defined in Section 2. Assume that $m>0$ and $\sigma$ is an irreducible generic cuspidal automorphic representation of $G_{n}(\mathbb{A})$. For $b>1$, the following formula holds:

$$
\begin{aligned}
& E_{a b, P_{a}}^{n, *}\left(\phi_{\Delta \otimes \sigma}, s\right)\left(\left(I_{a}, h\right)\right) \\
& =L\left(2 s+1, \tau, \rho^{(-)^{b+1}}\right) E_{a(b-1)}^{n-a, *}\left(\lambda_{-1 / 2}\left(i_{n-a}^{*} \phi_{Q}\right), s+\frac{1}{2}\right)(h) \\
& \quad+\frac{L\left(2 s, \tau, \rho^{(-)^{b+1}}\right)}{\varepsilon_{b}^{\prime}(s)} E_{a(b-1)}^{n-a, *}\left(\lambda_{1 / 2}\left(i_{n-a}^{*} \circ N(\omega, \cdot)\right) \tilde{\phi}, s-\frac{1}{2}\right)(h),
\end{aligned}
$$

where $\lambda_{-1 / 2}\left(i_{n-a}^{*} \phi_{Q}\right):=\lambda_{-1 / 2}\left(i_{n-a}^{*} \phi_{Q_{a, a(b-1)}^{a b}}\right)_{\Delta(\tau, b-1) \otimes \sigma}$.

3C. Normalization for the case of $\boldsymbol{m}=\mathbf{0}$. In this section, we consider the case of $m=0$. Due to the similarity between the cases of $m=0$ and $m>0$, we will just briefly sketch the result here. We continue to use the notation and references (which will not be mentioned) introduced in previous sections.

Note that when $m=0, G_{n}=\mathrm{SO}_{2 n}$ must be $F$-split. In this case, we divide the $G_{n}$ into two types: Type (1), $G_{n}=\mathrm{Sp}_{2 n}$ and $\mathrm{U}_{2 n+1}$, and Type (2), $G_{n}=\mathrm{SO}_{2 n+1}$, $\mathrm{SO}_{2 n}$, and $\mathrm{U}_{2 n}$.

In order to normalize the Eisenstein series, we consider the intertwining operator $M\left(\omega^{\prime},|\cdot|_{F^{\prime}}^{s} \Delta\right)(\phi)$ with

$$
\omega^{\prime}=(-1)^{a b}\left(\begin{array}{ll} 
& I_{a b} \\
\pm I_{a b} &
\end{array}\right)
$$

where the $I$ in the middle is the identity matrix of order at most one, that is, it either is 1 or disappears, depending on the structure of $G_{n}$.

For any factorizable function $\phi=\bigotimes_{v} \phi_{v}$, we write

$$
M\left(\omega^{\prime},|\cdot|_{F^{\prime}}^{s} \Delta\right)(\phi)=\prod_{v} M\left(\omega^{\prime},|\cdot|_{F_{v}^{\prime}}^{s} \Delta_{v}\right)\left(\phi_{v}\right)
$$

and for each local place $v$, define

$$
N^{\prime}\left(\omega^{\prime},|\cdot|_{F_{v}^{\prime}}^{s} \Delta\left(\tau_{v}, b\right)\right)\left(\phi_{v}\right)=\frac{1}{r^{\prime}\left(\omega^{\prime},|\cdot|_{F_{v}^{\prime}}^{s} \Delta\left(\tau_{v}, b\right)\right)} M\left(\omega^{\prime},|\cdot|_{F_{v}^{\prime}}^{s} \Delta\left(\tau_{v}, b\right)\right)\left(\phi_{v}\right),
$$

where the local normalizing factor $r^{\prime}\left(\omega^{\prime},|\cdot|_{F_{v}^{\prime}}^{s} \Delta\left(\tau_{v}, b\right)\right)$ is defined as follows. 
When $G_{n}$ is of Type (1), define

$$
\begin{aligned}
r^{\prime}\left(\omega^{\prime},|\cdot|_{F_{v}^{\prime}}^{s} \Delta\left(\tau_{v}, b\right)\right):=\frac{L\left(s, \Delta\left(\tau_{v}, b\right)\right)}{L\left(s+1, \Delta\left(\tau_{v}, b\right)\right) \varepsilon\left(s, \Delta\left(\tau_{v}, b\right), \psi_{v}\right)} \\
\quad \times \frac{L\left(2 s, \Delta\left(\tau_{v}, b\right), \rho\right)}{L\left(2 s+1, \Delta\left(\tau_{v}, b\right), \rho\right) \varepsilon\left(2 s, \Delta\left(\tau_{v}, b\right), \rho, \psi_{v}\right)} ;
\end{aligned}
$$

and when $G_{n}$ is of Type (2), define

$$
r^{\prime}\left(\omega^{\prime},|\cdot|_{F_{v}^{\prime}}^{s} \Delta\left(\tau_{v}, b\right)\right)=\frac{L\left(2 s, \Delta\left(\tau_{v}, b\right), \rho\right)}{L\left(2 s+1, \Delta\left(\tau_{v}, b\right), \rho\right) \varepsilon\left(2 s, \Delta\left(\tau_{v}, b\right), \rho, \psi_{v}\right)} .
$$

Then we define $r^{\prime}\left(\omega^{\prime},|\cdot|_{F^{\prime}}^{s} \Delta(\tau, b)\right)=\prod_{v} r^{\prime}\left(\omega^{\prime},|\cdot|_{F_{v}^{\prime}}^{s} \Delta\left(\tau_{v}, b\right)\right)$. The global normalized intertwining operator is

$$
N^{\prime}\left(\omega^{\prime},|\cdot|_{F^{\prime}}^{s} \Delta\right)=\prod_{v} N^{\prime}\left(\omega^{\prime},|\cdot|_{F_{v}^{\prime}}^{s} \Delta\left(\tau_{v}, b\right)\right) .
$$

We calculate the $L$-functions as in (3-2) and obtain

$$
\frac{L(s, \Delta(\tau, b))}{L(s+1, \Delta(\tau, b))}=\frac{L\left(s-\frac{b-1}{2}, \tau\right)}{L\left(s+\frac{b+1}{2}, \tau\right)}
$$

and

$$
\frac{L(2 s, \Delta(\tau, b), \rho)}{L(2 s+1, \Delta(\tau, b), \rho)}=\frac{\prod_{i=1}^{\lceil b / 2\rceil} L\left(f_{b, i}(s), \tau, \rho\right) \prod_{i=1}^{\lfloor b / 2\rfloor} L\left(f_{b, i}(s)+1, \tau, \rho^{-}\right)}{\prod_{i=1}^{\lceil b / 2\rceil} L\left(e_{b, i}(s)+1, \tau, \rho\right) \prod_{i=1}^{\lfloor b / 2\rfloor} L\left(e_{b, i}(s), \tau, \rho^{-}\right)},
$$

where $e_{b, i}(s):=2 s+b+1-2 i, f_{b, i}(s):=2 s-b-1+2 i$.

When $G_{n}$ is of Type (1), define

$$
\begin{aligned}
\alpha_{b}(s) & =\prod_{i=1}^{\lceil b / 2\rceil} L\left(f_{b, i}(s), \tau, \rho\right) \prod_{i=1}^{\lfloor b / 2\rfloor} L\left(f_{b, i}(s)+1, \tau, \rho^{-}\right) L\left(s-\frac{b-1}{2}, \tau\right), \\
\beta_{b}(s) & =\prod_{i=1}^{\lceil b / 2\rceil} L\left(e_{b, i}(s)+1, \tau, \rho\right) \prod_{i=1}^{\lfloor b / 2\rfloor} L\left(e_{b, i}(s), \tau, \rho^{-}\right) L\left(s+\frac{b+1}{2}, \tau\right), \\
\varepsilon_{b}(s) & =\prod_{i=1}^{\lceil b / 2\rceil} \varepsilon\left(f_{b, i}(s), \tau, \rho\right) \prod_{i=1}^{\lfloor b / 2\rfloor} \varepsilon\left(f_{b, i}(s)+1, \tau, \rho^{-}\right) \varepsilon\left(s-\frac{b-1}{2}, \tau\right),
\end{aligned}
$$

and then define

$$
N\left(\omega^{\prime},|\cdot|_{F^{\prime}}^{s} \Delta\right)=\frac{\varepsilon_{b}(s) N^{\prime}\left(\omega^{\prime},|\cdot|_{F^{\prime}}^{s} \Delta\right)}{\varepsilon(s, \Delta(\tau, b), \psi) \varepsilon(2 s, \Delta(\tau, b), \rho, \psi)} .
$$


When $G_{n}$ is of Type (2), define

$$
\begin{aligned}
& \alpha_{b}(s)=\prod_{i=1}^{\lceil b / 2\rceil} L\left(f_{b, i}(s), \tau, \rho\right) \prod_{i=1}^{\lfloor b / 2\rfloor} L\left(f_{b, i}(s)+1, \tau, \rho^{-}\right), \\
& \beta_{b}(s)=\prod_{i=1}^{\lceil b / 2\rceil} L\left(e_{b, i}(s)+1, \tau, \rho\right) \prod_{i=1}^{\lfloor b / 2\rfloor} L\left(e_{b, i}(s), \tau, \rho^{-}\right), \\
& \varepsilon_{b}(s)=\prod_{i=1}^{\lceil b / 2\rceil} \varepsilon\left(f_{b, i}(s), \tau, \rho\right) \prod_{i=1}^{\lfloor b / 2\rfloor} \varepsilon\left(f_{b, i}(s)+1, \tau, \rho^{-}\right),
\end{aligned}
$$

and then define

$$
N\left(\omega^{\prime},|\cdot|_{F^{\prime}}^{s} \Delta\right)=\frac{\varepsilon_{b}(s) N^{\prime}\left(\omega^{\prime},|\cdot|_{F^{\prime}}^{s} \Delta\right)}{\varepsilon(2 s, \Delta(\tau, b), \rho, \psi)} .
$$

Now we define the normalizing factor by

$$
r\left(\omega^{\prime},|\cdot|_{F^{\prime}}^{s} \Delta\right):=\frac{\alpha_{b}(s)}{\beta_{b}(s) \varepsilon_{b}(s)} .
$$

Then

$$
N\left(\omega^{\prime},|\cdot|_{F^{\prime}}^{s} \Delta(\tau, b)\right)=\frac{1}{r\left(\omega^{\prime},|\cdot|_{F^{\prime}}^{s} \Delta(\tau, b)\right)} M\left(\omega^{\prime},|\cdot|_{F^{\prime}}^{s} \Delta(\tau, b)\right) .
$$

Remark 3.3. The terms $\alpha_{b}(s)$ and $\beta_{b}(s)$ correspond to the terms $a_{b}(s)$ and $b_{b}(s)$ in [Brenner 2009, Section 4.2]. We correct the definition of $b_{b}(s)$ in [Brenner 2009] here.

We use $\beta_{b}(s)$ to normalize the Eisenstein series

$$
E_{a b}^{n, *}\left(\phi_{\Delta(\tau, b)}, s\right):=\beta_{b}(s) E_{a b}^{n}\left(\phi_{\Delta(\tau, b)}, s\right) .
$$

Then, similarly to (3-6), we have the functional equation for the normalized Eisenstein series:

$$
E_{a b}^{n, *}\left(\phi_{\Delta(\tau, b)}, s\right)=E_{a b}^{n, *}\left(N\left(\omega^{\prime},|\cdot|_{F^{\prime}}^{s} \Delta\right)(\phi),-s\right) .
$$

Next, we normalize the intertwining operator as defined in (2-7),

$$
M(\omega, \cdot):=M\left(\omega,|\cdot|_{F^{\prime}}^{s-1 / 2} \Delta(\tau, b-1) \otimes|\cdot|_{F^{\prime}}^{s+(b-1) / 2} \tau\right),
$$

by

$$
N(\omega, \cdot):=N\left(\omega,|\cdot|_{F^{\prime}}^{s-1 / 2} \Delta(\tau, b-1) \otimes|\cdot|_{F^{\prime}}^{s+(b-1) / 2} \tau\right)=\frac{M(\omega, \cdot)}{r(\omega, \cdot)}
$$


with $r(\omega, \cdot)$ defined as follows. When $G_{n}$ is of Type (1), define

$$
\begin{aligned}
r(\omega, \cdot)= & r\left(\omega,|\cdot|_{F^{\prime}}^{s-1 / 2} \Delta(\tau, b-1) \otimes|\cdot|_{F^{\prime}}^{s+(b-1) / 2} \tau\right) \\
= & \frac{L\left(s+\frac{b-1}{2}, \tau\right) L(2 s+b-1, \tau, \rho) L\left(2 s+\frac{b}{2}-1, \Delta(\tau, b-1) \times \tau^{*}\right)}{L\left(s+\frac{b+1}{2}, \tau\right) L(2 s+b, \tau, \rho) L\left(2 s+\frac{b}{2}, \Delta(\tau, b-1) \times \tau^{*}\right)} \\
& \times \frac{1}{\varepsilon\left(s+\frac{b-1}{2}, \tau\right) \varepsilon(2 s+b-1, \tau, \rho) \varepsilon\left(2 s+\frac{b}{2}-1, \Delta(\tau, b-1) \times \tau^{*}\right)} ;
\end{aligned}
$$

and when $G_{n}$ is of Type (2), define

$$
\begin{aligned}
r(\omega, \cdot)= & r\left(\omega,|\cdot|_{F^{\prime}}^{s-1 / 2} \Delta(\tau, b-1) \otimes|\cdot|_{F^{\prime}}^{s+(b-1) / 2} \tau\right) \\
= & \frac{L(2 s+b-1, \tau, \rho) L\left(2 s+\frac{b}{2}-1, \Delta(\tau, b-1) \times \tau^{*}\right)}{L(2 s+b, \tau, \rho) L\left(2 s+\frac{b}{2}, \Delta(\tau, b-1) \times \tau^{*}\right)} \\
& \times \frac{1}{\varepsilon(2 s+b-1, \tau, \rho) \varepsilon\left(2 s+\frac{b}{2}-1, \Delta(\tau, b-1) \times \tau^{*}\right)},
\end{aligned}
$$

where $\tau^{*}=\tau$ if $F^{\prime}=F$ and $\tau^{*}=\tau^{\iota}$ if $F^{\prime}=E$, with $\iota$ being the nontrivial element in the Galois group $\Gamma_{E / F}$.

The following, corresponding to Proposition 3.1, is also true when $m=0$.

Proposition 3.4. For $\operatorname{Re}(s)>0$ and $b>1$, the normalized global intertwining operator $N\left(\omega,|\cdot|_{F^{\prime}}^{s-1 / 2} \Delta(\tau, b-1) \otimes|\cdot|_{F^{\prime}}^{s+(b-1) / 2} \tau\right)$ is holomorphic for all choices of data, and nonzero for some choice of data.

The proof follows from that of Proposition 3.1, and we omit the details here.

By substituting the normalized Eisenstein series $E_{a b}^{n, *}\left(\phi_{\Delta(\tau, b)}, s\right)$ in (3-9) and the normalized intertwining operator $N(\omega, \cdot)$ in (3-11) into the induction formula (2-9) in Proposition 2.3, we obtain

$$
\begin{aligned}
& E_{a b, P_{a}}^{n, *}\left(\phi_{\Delta}, s\right)\left(\left(I_{a}, h\right)\right) \\
& =\frac{\beta_{b}(s)}{\beta_{b-1}\left(s+\frac{1}{2}\right)} E_{a(b-1)}^{n-a, *}\left(\lambda_{-1 / 2}\left(i_{n-a}^{*} \phi_{Q_{a, a(b-1)}^{a b}}\right) \Delta(\tau, b-1), s+\frac{1}{2}\right)(h) \\
& \quad+\frac{\beta_{b}(s) \cdot r(\omega, \cdot)}{\beta_{b-1}\left(s-\frac{1}{2}\right)} E_{a(b-1)}^{n-a, *}\left(\lambda_{1 / 2}\left(i_{n-a}^{*} \circ N(\omega, \cdot)\right) \tilde{\phi}, s-\frac{1}{2}\right)(h) .
\end{aligned}
$$

Using a similar calculation as in (3-2), it is easy to verify that 


$$
\begin{aligned}
\frac{\beta_{b}(s)}{\beta_{b-1}\left(s+\frac{1}{2}\right)} & =L\left(2 s+1, \tau, \rho^{(-)^{b+1}}\right), \\
\frac{\beta_{b}(s)}{\beta_{b-1}\left(s-\frac{1}{2}\right)} r(\omega, \cdot) & =\frac{L\left(2 s, \tau, \rho^{(-)^{b+1}}\right)}{\varepsilon_{b}^{\prime}(s)},
\end{aligned}
$$

where $\varepsilon_{b}^{\prime}(s)$ is defined as follows. When $G_{n}$ is of Type (1), define $\varepsilon_{b}^{\prime}(s)$ to be the product

$$
\varepsilon(2 s+b-1, \tau, \rho) \varepsilon\left(2 s+\frac{b}{2}-1, \Delta(\tau, b-1) \times \tau^{*}\right) \varepsilon\left(s+\frac{b-1}{2}, \tau\right) ;
$$

and when $G_{n}$ is of Type (2), define

$$
\varepsilon_{b}^{\prime}(s)=\varepsilon(2 s+b-1, \tau, \rho) \varepsilon\left(2 s+\frac{b}{2}-1, \Delta(\tau, b-1) \times \tau^{*}\right) .
$$

Therefore, for $b>1$, we obtain the following normalized induction formula, which is similar to Proposition 3.2.

Proposition 3.5. With notation as defined above, for $b>1$, the following formula holds:

$$
\begin{aligned}
& E_{a b, P_{a}}^{n, *}\left(\phi_{\Delta}, s\right)\left(\left(I_{a}, h\right)\right) \\
& =L\left(2 s+1, \tau, \rho^{(-)^{b+1}}\right) E_{a(b-1)}^{n-a, *}\left(\lambda_{-1 / 2}\left(i_{n-a}^{*} \phi_{Q}\right), s+\frac{1}{2}\right)(h) \\
& \quad+\frac{L\left(2 s, \tau, \rho^{(-)^{b+1}}\right)}{\varepsilon_{b}^{\prime}(s)} E_{a(b-1)}^{n-a, *}\left(\lambda_{1 / 2}\left(i_{n-a}^{*} \circ N(\omega, \cdot)\right) \tilde{\phi}, s-\frac{1}{2}\right)(h),
\end{aligned}
$$

where $\lambda_{-1 / 2}\left(i_{n-a}^{*} \phi_{Q}\right):=\lambda_{-1 / 2}\left(i_{n-a}^{*} \phi_{Q_{a, a(b-1)}^{a b}}\right)_{\Delta(\tau, b-1)}$.

\section{Proof of Theorem $1.2(m>0)$}

We are going to prove Theorem 1.2 for the case where $m>0$ using the normalized induction formula given in Proposition 3.2. From now on, we only consider symplectic group and $F$-quasisplit special orthogonal group cases.

4A. Case of $\boldsymbol{b}=1$. The case of $b=1$ is the starting step of our proof by induction. Assume that $s \in \mathbb{C}$ with $\operatorname{Re}(s)>0$.

By Equation (3-5), we normalize $E_{a}^{n}\left(\phi_{\tau \otimes \sigma}, s\right)$ as follows:

$$
E_{a}^{n, *}\left(\phi_{\tau \otimes \sigma}, s\right)=L(s+1, \tau \times \sigma) L(2 s+1, \tau, \rho) E_{a}^{n}\left(\phi_{\tau \otimes \sigma}, s\right) .
$$

By [Mœglin and Waldspurger 1995, Proposition II.1.7], the constant term of the Eisenstein series $E_{a}^{n}\left(\phi_{\tau \otimes \sigma}, s\right)$ along a standard parabolic subgroup $P^{\prime}$ is always zero unless $P^{\prime}=P_{a}$. In the case of $P^{\prime}=P_{a}$, we have

$$
E_{a, P_{a}}^{n}\left(\phi_{\tau \otimes \sigma}, s\right)=\lambda_{s} \phi_{\tau \otimes \sigma}(g)+M\left(\omega^{\prime},|\cdot|_{F^{\prime}}^{s} \tau \otimes \sigma\right)\left(\lambda_{s} \phi\right) .
$$


By Lemma I.4.10 of [Mœglin and Waldspurger 1995], the Eisenstein series $E_{a}^{n}\left(\phi_{\tau \otimes \sigma}, s\right)$ has a pole at some point $s_{0}$ if and only if the constant term of $E_{a, P_{a}}^{n}\left(\phi_{\tau \otimes \sigma}, s\right)$ has a pole at $s_{0}$, and hence if and only if the term

$$
M\left(\omega^{\prime},|\cdot|_{F^{\prime}}^{s} \tau \otimes \sigma\right)\left(\lambda_{s} \phi\right)
$$

has a pole at $s_{0}$, since the first term $\lambda_{s} \phi_{\tau \otimes \sigma}(g)$ is holomorphic. By our normalization, we have

$$
M\left(\omega^{\prime},|\cdot|_{F^{\prime}}^{s} \tau \otimes \sigma\right)\left(\lambda_{s} \phi\right)=r\left(\omega^{\prime},|\cdot|_{F^{\prime}}^{s} \tau \otimes \sigma\right) N\left(\omega^{\prime},|\cdot|_{F^{\prime}}^{s} \tau \otimes \sigma\right)(\phi),
$$

and for $\operatorname{Re}(s)>0$, by [Cogdell et al. 2004, Theorem 11.1], the normalized global intertwining operator $N\left(\omega^{\prime},|\cdot|_{F^{\prime}}^{s} \tau \otimes \sigma\right)$ is holomorphic for all choice of data and nonzero for some choice of data. Thus, it reduces to checking the existence of the pole at $s=s_{0}$ of the global normalizing factor $r\left(\omega^{\prime},|\cdot|_{F^{\prime}}^{s} \tau \otimes \sigma\right)$.

Recall from (3-3) that the global normalizing factor $r\left(\omega^{\prime},|\cdot|_{F^{\prime}}^{s} \tau \otimes \sigma\right)$ in this case is

$$
\frac{L(s, \tau \times \sigma) L(2 s, \tau, \rho)}{L(s+1, \tau \times \sigma) L(2 s+1, \tau, \rho) \varepsilon(s, \tau \times \sigma) \varepsilon(2 s, \tau, \rho)} .
$$

Since both $\varepsilon(s, \tau \times \sigma)$ and $\varepsilon(2 s, \tau, \rho)$ are holomorphic and nonzero, the poles of the global normalizing factor $r\left(\omega^{\prime},|\cdot|_{F^{\prime}}^{s} \tau \otimes \sigma\right)$ at $s=s_{0}>0$ with $\operatorname{Re}\left(s_{0}\right)>0$ are the same as the poles of the quotient

$$
\frac{L(s, \tau \times \sigma) L(2 s, \tau, \rho)}{L(s+1, \tau \times \sigma) L(2 s+1, \tau, \rho)}
$$

at $s=s_{0}>0$ with $\operatorname{Re}\left(s_{0}\right)>0$.

Since $\sigma$ is generic and $\tau$ is self-dual, by the global Langlands functorial transfer from $G_{n}$ to a general linear group [Cogdell et al. 2004] and the analytic property of the complete $L$-functions of the Rankin-Selberg convolution [Cogdell and PiatetskiShapiro 2004; Mœglin and Waldspurger 1989], we deduce that the complete $L$ function $L(s, \tau \times \sigma)$ is holomorphic at all $s \in \mathbb{C}$ except for a possible simple pole at $s=0$ or 1 , and is nonzero when $\operatorname{Re}(s) \leq 0$ or $\operatorname{Re}(s) \geq 1$. Such a pole occurs if and only if $\tau$ occurs as an isobaric summand in the image of $\sigma$ under the Langlands functorial transfer [Cogdell et al. 2004].

On the other hand, by [Grbac 2011], based on the work of Arthur [2013] on the classification of the discrete spectrum of $G_{n}(\mathbb{A})$, the complete $L$-function $L(s, \tau, \rho)$ is holomorphic at all $s \in \mathbb{C}$ except for a possible simple pole at $s=0$ or 1 , and is nonzero when $\operatorname{Re}(s) \leq 0$ or $\operatorname{Re}(s) \geq 1$. Such a pole occurs if and only if $\tau$ can descend to an irreducible generic cuspidal automorphic representation of a classical group determined by $\rho$ [Ginzburg et al. 2011].

Hence, when $\operatorname{Re}(s)>0$, the denominator $L(s+1, \tau \times \sigma) L(2 s+1, \tau, \rho)$ is holomorphic and nonzero, and the numerator $L(s, \tau \times \sigma) L(2 s, \tau, \rho)$ is holomorphic 
except for a possible simple pole at $s=\frac{1}{2}$ or $s=1$. This proves the theorem for the case of $b=1$. We summarize the above as the following.

Proposition 4.1 (case $b=1$ of Theorem 1.2). Let $G_{n}$ be the symplectic group or the F-quasisplit special orthogonal group. Let $\tau$ be an irreducible unitary cuspidal automorphic representation of $\mathrm{GL}_{a}(\mathbb{A})$ and let $\sigma$ be an irreducible generic cuspidal automorphic representation of $G_{m}(\mathbb{A})$. The normalized Eisenstein series $E_{a}^{n, *}\left(\phi_{\tau \otimes \sigma}, s\right)$ is holomorphic at $\operatorname{Re}(s) \geq 0$, except at $s=\frac{1}{2}$ and $s=1$, where it has possible simple poles. Moreover:

(1) $E_{a}^{n, *}\left(\phi_{\tau \otimes \sigma}, s\right)$ has a simple pole at $s=\frac{1}{2}$ if and only if $L(s, \tau, \rho)$ has a pole at $s=1$, and $L\left(\frac{1}{2}, \tau \times \sigma\right) \neq 0$.

(2) $E_{a}^{n, *}\left(\phi_{\tau \otimes \sigma}, s\right)$ has a simple pole at $s=1$ if and only if $L(s, \tau \times \sigma)$ has a pole at $s=1$.

In particular, $E_{a}^{n, *}\left(\phi_{\tau \otimes \sigma}, s\right)$ is holomorphic at $\operatorname{Re}(s)>0$ if $\tau$ is not self-dual.

Proposition 4.1 includes the case of $m=0$, which is proved in [Grbac 2011].

We remark that by the functional equation for the normalized Eisenstein series (3-6), one deduces the analytic properties at $\operatorname{Re}(s)<0$, since when $b=1$, the normalized intertwining operator occurring in the functional equation is holomorphic for $\operatorname{Re}(s)>0$ and is a nonzero operator. At $\operatorname{Re}(s)=0$, it is holomorphic (Corollary 4.3).

4B. Case of $\boldsymbol{b}>1$. This general case of Theorem 1.2 is proved by using the normalized induction formula (Proposition 3.2) and the case of $b=1$ (Proposition 4.1). One technical point is to prove that the normalized Eisenstein series $E_{a b}^{n, *}\left(\phi_{\tau \otimes \sigma}, s\right)$ is holomorphic at $s=0$ (Corollary 4.3), which is a consequence of the following.

Proposition 4.2. Let $G_{n}$ be the symplectic group or the F-quasisplit special orthogonal group. Assume that $\sigma$ is an irreducible generic (or tempered if nongeneric) cuspidal automorphic representation of $G_{m}(\mathbb{A})$. If $\beta_{b}(s)$ has a pole at $s=0$, then the pole at $s=0$ of $\beta_{b}(s)$ must be simple and $E_{a b}^{n}\left(\phi_{\Delta(\tau, b) \otimes \sigma}, s\right)$ must vanish at $s=0$.

Proof. Note first that by [Arthur 2013], the Langlands-Shahidi normalization works for intertwining operators with tempered induced data at $s=0$. Hence we allow here that $\sigma$ could be any irreducible tempered cuspidal automorphic representation if it is not generic.

Assume that $\beta_{b}(s)$, as defined in (1-1) or in Section 3A with more detail, has a pole at $s=0$. It implies that when $b=1$,

$$
\beta_{1}(s)=L(2 s+1, \tau, \rho) L(s+1, \tau \times \sigma)
$$


has a pole at $s=0$, and when $b>1$, the only factor in $\beta_{b}(s)$ to have a possible pole at $s=0$ is $L\left(s, \tau, \rho^{(-)^{b+1}}\right)$.

When $b=1, L(2 s+1, \tau, \rho)$ and $L(s+1, \tau \times \sigma)$ both have at most a simple pole at $s=0$, but they cannot happen at the same time, since $L(s+1, \tau \times \sigma)$ having a simple pole at $s=0$ implies that $L\left(s, \tau, \rho^{-}\right)$has a pole at $s=1$. Therefore, $\beta_{1}(s)$ has at most a simple pole at $s=0$. When $b>1, L\left(s, \tau, \rho^{(-)^{b+1}}\right)$ has at most a simple pole at $s=0$. So, for $b>1, \beta_{b}(s)$ also has at most a simple pole at $s=0$. Hence, $\beta_{b}(s)$ has at most a simple pole at $s=0$ for all $b \geq 1$. Now the assumption that $\beta_{b}(s)$ has a pole at $s=0$ implies that $\operatorname{ord}_{s=0}\left(\beta_{b}(s)\right)=1$ for any $b \geq 1$, that is, $\beta_{b}(s)$ has a simple pole at $s=0$.

By the functional equation (3-1) and the normalized functional equation (3-6), we have

$$
E_{a b}^{n}\left(\phi_{\Delta \otimes \sigma}, s\right)=r\left(\omega^{\prime},|\cdot|_{F}^{s} \Delta \otimes \sigma\right) E_{a b}^{n}\left(N\left(\omega^{\prime},|\cdot|_{F}^{s} \Delta \otimes \sigma\right)(\phi),-s\right),
$$

with

$$
r\left(\omega^{\prime},|\cdot|_{F}^{s} \Delta \otimes \sigma\right)=\frac{\beta_{b}(-s)}{\beta_{b}(s)} .
$$

By the above discussion on the pole at $s=0$ of $\beta_{b}(s)$, it is clear that

$$
\left.r\left(\omega^{\prime},|\cdot|_{F}^{s} \Delta \otimes \sigma\right)\right|_{s=0}=(-1)^{\operatorname{ord}_{s=0}\left(\beta_{b}(s)\right)}=-1,
$$

and hence

$$
\left.E_{a b}^{n}\left(\phi_{\Delta \otimes \sigma}, s\right)\right|_{s=0}=-\left.E_{a b}^{n}\left(N\left(\omega^{\prime},|\cdot|_{F}^{s} \Delta \otimes \sigma\right)(\phi),-s\right)\right|_{s=0} .
$$

So it suffices to show that the normalized intertwining operator $N\left(\omega^{\prime},|\cdot|_{F}^{S} \Delta \otimes \sigma\right)$ is an identity map at $s=0$. We deduce this fact from the work of Arthur.

Arthur [2013, Corollary 7.3.5] proved that for the tempered or generic representation that has the Arthur parameter such that $\beta_{b}(s)$ has a simple pole at $s=0$, the normalized intertwining operator at $s=0$ has the identity

$$
\lambda\left(\omega^{\prime}\right) \iota\left(\omega^{\prime}\right) \circ \mathcal{N}\left(\omega^{\prime}, \Delta \otimes \sigma\right)=\mathrm{Id},
$$

where $\lambda\left(\omega^{\prime}\right)$ is the $\lambda$-factor (see for example [Keys and Shahidi 1988, Section 2]), $\iota\left(\omega^{\prime}\right)$ is a canonical map from $\omega^{\prime} \Delta(\tau, b) \otimes \sigma$ to $\Delta(\tau, b) \otimes \sigma$ defined by Arthur [2013], and $\mathcal{N}\left(\omega^{\prime}, \Delta \otimes \sigma\right)$ is the evaluation at $s=0$ of the normalized intertwining operator from the induced representation $\mathrm{I}(\Delta \otimes \sigma, s)$ to $\mathrm{I}\left(\omega^{\prime} \Delta \otimes \sigma,-s\right)$ (the vector-valued induced representations). The intertwining operator $N\left(\omega^{\prime},|\cdot|_{F}^{s} \Delta \otimes \sigma\right)$ considered in this paper is a map from the space of automorphic forms

$$
A\left(N_{a b}(\mathbb{A}) M_{a b}(F) \backslash G(\mathbb{A})\right)_{\left.|\cdot|\right|_{F} ^{s} \Delta \otimes \sigma}
$$

to the space

$$
A\left(N_{a b}(\mathbb{A}) M_{a b}(F) \backslash G(\mathbb{A})\right)_{|\cdot|_{F}^{-s} \omega^{\prime} \Delta \otimes \sigma}
$$


Note that by the strong multiplicity one theorem for $\mathrm{GL}_{n}$ and the definition of the isotypic component $A\left(N_{a b}(\mathbb{A}) M_{a b}(F) \backslash G(\mathbb{A})\right)_{|\cdot|_{F}^{s} \Delta \otimes \sigma}$, this subspace depends only on the equivalence class of $\Delta$, but not on its realization $\Delta$ in the space of automorphic forms. Therefore, we have the following relation between the two versions of the normalized intertwining operators at $s=0$ :

$$
N\left(\omega^{\prime}, \Delta \otimes \sigma\right)=\iota\left(\omega^{\prime}\right) \circ \mathcal{N}\left(\omega^{\prime}, \Delta \otimes \sigma\right) .
$$

Since the global $\lambda$-factor is trivial (see [Keys and Shahidi 1988, Section 2]), we have the following identity at $s=0$ :

$$
E_{a b}^{n}\left(N\left(\omega^{\prime},|\cdot|_{F}^{s} \Delta \otimes \sigma\right)(\phi), 0\right)=E_{a b}^{n}\left(\phi_{\Delta(\tau, b) \otimes \sigma}, 0\right) .
$$

By comparing with the identity (4-1), we obtain that $E_{a b}^{n}\left(\phi_{\Delta(\tau, b) \otimes \sigma}, s\right)$ vanishes at $s=0$. This completes the proof.

Following from the definition of the normalized Eisenstein series, we have:

Corollary 4.3. Let $G_{n}$ be the symplectic group or the F-quasisplit special orthogonal group. Assume that $\sigma$ is an irreducible generic (or tempered if nongeneric) cuspidal automorphic representation of $G_{m}(\mathbb{A})$. The normalized Eisenstein series $E_{a b}^{n, *}\left(\phi_{\Delta(\tau, b) \otimes \sigma}, s\right)$ is holomorphic at the point $s=0$.

By using Corollary 4.3 and the normalized induction formula in Proposition 3.2, we are able to prove Theorem 1.2 for the case of $b>1$, that is, to determine the location of possible poles of the normalized Eisenstein series $E_{a b}^{n, *}\left(\phi_{\Delta(\tau, b) \otimes \sigma}, s\right)$ for $b>1$. To do so, we consider the following four cases:

(1) $L(s, \tau, \rho)$ has a pole at $s=1$, and $L\left(\frac{1}{2}, \tau \times \sigma\right) \neq 0$;

(2) $L(s, \tau, \rho)$ has a pole at $s=1$, and $L\left(\frac{1}{2}, \tau \times \sigma\right)=0$;

(3) $L\left(s, \tau, \rho^{-}\right)$has a pole at $s=1$, and $L(s, \tau \times \sigma)$ has a pole at $s=1$;

(4) $L\left(s, \tau, \rho^{-}\right)$has a pole at $s=1$, and $L(s, \tau \times \sigma)$ is holomorphic at $s=1$.

We define the sets of possible poles according to the four cases:

$$
X_{b, \tau, \sigma}^{+}:= \begin{cases}\left\{\hat{0}, \ldots, \frac{b-2}{2}, \frac{b}{2}\right\} & \text { in Case (1); } \\ \left\{\hat{0}, \ldots, \frac{b-4}{2}, \frac{b-2}{2}\right\} & \text { in Case (2); } \\ \left\{\hat{0}, \ldots, \frac{b-1}{2}, \frac{b+1}{2}\right\} & \text { in Case (3); } \\ \left\{\hat{0}, \ldots, \frac{b-3}{2}, \frac{b-1}{2}\right\} & \text { in Case (4) }\end{cases}
$$

When $b=1$, the set $X_{1, \tau, \sigma}^{+}$is equal to the set $\left\{\frac{1}{2}\right\}$ in Case (1); is empty in Case (2); is equal to the set $\{1\}$ in Case (3); and is empty in Case (4). Hence the set $X_{b, \tau, \sigma}^{+}$is 
the set of possible poles of the normalized Eisenstein series $E_{a b}^{n, *}\left(\phi_{\Delta(\tau, b) \otimes \sigma}, s\right)$ for $b=1$ and $\operatorname{Re}(s)>0$, by Proposition 4.1.

It is clear that when $b=2$, the set $X_{b, \tau, \sigma}^{+}$is also empty in Case (2). Note that we omit 0 in the set $X_{b, \tau, \sigma}^{+}$, since the normalized Eisenstein series $E_{a b}^{n, *}\left(\phi_{\Delta(\tau, b) \otimes \sigma}, s\right)$ is holomorphic at $s=0$ (Corollary 4.3).

Here is the case of $b>1$ and $m>0$ of Theorem 1.2. The proof of this theorem for $\operatorname{Re}(s) \geq \frac{1}{2}$ is given by an induction argument, while the proof of this theorem for $0<\operatorname{Re}(s)<\frac{1}{2}$ needs the Arthur classification [2013] of the discrete spectrum, which is stated here and will be proved in Section 6C.

Proposition 4.4 (case $0<\operatorname{Re}(s)<\frac{1}{2}$ of Theorem 1.2). Let $G_{n}$ be the symplectic group or the F-quasisplit special orthogonal group. Assume that the irreducible cuspidal automorphic representation $\sigma$ of $G_{m}(\mathbb{A})$ is generic and the irreducible unitary cuspidal automorphic representation $\tau$ of $\mathrm{GL}_{a}(\mathbb{A})$ is self-dual. Then $E_{a b}^{n, *}\left(\phi_{\Delta(\tau, b) \otimes \sigma}, s\right)$ is holomorphic for $0<\operatorname{Re}(s)<\frac{1}{2}$.

With Propositions 4.1 and 4.4, Corollary 4.3, and the normalized induction formula (3-8), we are able to prove the following.

Theorem 4.5 (case $b>1$ and $m>0$ of Theorem 1.2). Let $G_{n}$ be the symplectic group or the F-quasisplit special orthogonal group. Assume that the irreducible cuspidal automorphic representation $\sigma$ of $G_{m}(\mathbb{A})$ is generic and the irreducible unitary cuspidal automorphic representation $\tau$ of $\mathrm{GL}_{a}(\mathbb{A})$ is self-dual. Then $E_{a b}^{n, *}\left(\phi_{\Delta(\tau, b) \otimes \sigma}, s\right)$ is holomorphic for $\operatorname{Re}(s) \geq 0$ except at $s=s_{0} \in X_{b, \tau, \sigma}^{+}$, where it may have possibly at most simple poles.

Proof. By Corollary 4.3 and Proposition $4.4, E_{a b}^{n, *}\left(\phi_{\Delta(\tau, b) \otimes \sigma}, s\right)$ is holomorphic at $0 \leq \operatorname{Re}(s)<\frac{1}{2}$, and hence we assume that $\operatorname{Re}(s) \geq \frac{1}{2}$ in the following discussion.

When $b=1$, it is Proposition 4.1. We may assume that $b>1$ and use the normalized induction formula (3-8):

$$
\begin{aligned}
& E_{a b, P_{a}}^{n, *}\left(\phi_{\Delta \otimes \sigma}, s\right)\left(\left(I_{a}, h\right)\right) \\
& =L\left(2 s+1, \tau, \rho^{\left.(-)^{b+1}\right)} E_{a(b-1)}^{n-a, *}\left(\lambda_{-1 / 2}\left(i_{n-a}^{*} \phi_{Q}\right), s+\frac{1}{2}\right)(h)\right. \\
& \quad+\frac{L\left(2 s, \tau, \rho^{(-)^{b+1}}\right)}{\varepsilon_{b}^{\prime}(s)} E_{a(b-1)}^{n-a, *}\left(\lambda_{1 / 2}\left(i_{n-a}^{*} \circ N(\omega, \cdot)\right) \tilde{\phi}, s-\frac{1}{2}\right)(h) .
\end{aligned}
$$

When $\operatorname{Re}(s) \geq \frac{1}{2}$, the term

$$
L\left(2 s+1, \tau, \rho^{(-)^{b+1}}\right) E_{a(b-1)}^{n-a, *}\left(\lambda_{-1 / 2}\left(i_{n-a}^{*} \phi_{Q}\right), s+\frac{1}{2}\right)(h)
$$

is holomorphic except for possible simple poles at $s_{0} \in X_{b-1, \tau, \sigma}^{+}+\frac{1}{2}$, by the induction assumption. 
The term

$$
\frac{L\left(2 s, \tau, \rho^{(-)^{b+1}}\right)}{\varepsilon_{b}^{\prime}(s)} E_{a(b-1)}^{n-a, *}\left(\lambda_{1 / 2}\left(i_{n-a}^{*} \circ N(\omega, \cdot)\right) \tilde{\phi}, s-\frac{1}{2}\right)(h)
$$

is holomorphic for $\operatorname{Re}(s) \geq 1$ except for possible simple poles at $X_{b-1, \tau, \sigma}^{+}-\frac{1}{2}$, by the induction assumption, while at $\frac{1}{2}<\operatorname{Re}(s)<1$, it is holomorphic by Proposition 4.4. At $s=\frac{1}{2}$,

$$
E_{a(b-1)}^{n-a, *}\left(\lambda_{1 / 2}\left(i_{n-a}^{*} \circ N(\omega, \cdot)\right) \tilde{\phi}, s-\frac{1}{2}\right)(h)
$$

is holomorphic by Corollary 4.3 , while the $L$-function $L\left(2 s, \tau, \rho^{(-)^{b+1}}\right)$ may have a simple pole according to the classification of four cases on the parity of $b$, the type of $\tau$, and the type of $G_{n}$ in the Introduction.

Hence $E_{a b}^{n, *}\left(\phi_{\Delta(\tau, b) \otimes \sigma}, s\right)$ is holomorphic for $\operatorname{Re}(s) \geq \frac{1}{2}$ except for possible simple poles at $s_{0} \in\left(X_{b-1, \tau, \sigma}^{+}+\frac{1}{2}\right) \cup\left(X_{b-1, \tau, \sigma}^{+}-\frac{1}{2}\right)$ with $\operatorname{Re}\left(s_{0}\right) \geq \frac{1}{2}$. It is easy to check that

$$
X_{b, \tau, \sigma}^{+}=\left(X_{b-1, \tau, \sigma}^{+}+\frac{1}{2}\right) \cup\left[\left(X_{b-1, \tau, \sigma}^{+}-\frac{1}{2}\right) \backslash\{0\}\right] .
$$

The theorem follows.

This completes the proof of Theorem 1.2 for the case of $m>0$. We conclude this section with the following remarks.

(1) Theorem 1.2 holds for the $F$-quasisplit unitary groups if Corollary 4.3 is proven for the $F$-quasisplit unitary groups, which is done since Arthur's work has been extended to the $F$-quasisplit unitary groups [Mok 2012]. The extension of Arthur's classification of the discrete spectrum for $F$-quasisplit unitary groups will also imply that the complete Asai (and twisted Asai) $L$-functions are holomorphic in $0<s<1$ (as in [Grbac 2011] for symplectic or $F$-split special orthogonal groups), which is one of the key ingredients in the proof of Theorem 1.2 for $b=1$ and $m>0$.

(2) Theorem 1.2 is also expected to hold when $\sigma$ is nongeneric, but tempered. The technical issue is the normalization of the local intertwining operators at all local places. At $p$-adic local fields, one can use Mœglin's work [2008; 2010]. Since her work at archimedean local places is not general enough to cover our cases, one needs more work, which will be considered in our future work.

(3) The current version of Theorem 1.2 is sufficient for our applications to the constructions of endoscopy correspondences considered in [Jiang 2011; 2012].

\section{Proof of Theorem $1.2(m=0)$}

In this case ( $m=0), G_{n}$ is either a symplectic group or an $F$-split special orthogonal group. When $b=1$, Theorem 1.2 for $m=0$ is given in [Grbac 2011, Theorem 3.1]. 
For $b>1$, the proof of case $m=0$ requires analogous results to Proposition 4.2 and Corollary 4.3, which are stated below. By the definition of $\beta_{b}(s)$ in this case as in Section 3C, the same proof works here.

Proposition 5.1. Let $G_{n}$ be a symplectic group or an $F$-split special orthogonal group. Assume that $b>1$ and $m=0$. If $\beta_{b}(s)$ has a pole at $s=0$, then $E_{a b}^{n}\left(\phi_{\Delta(\tau, b)}, s\right)$ vanishes at $s=0$. Moreover, the normalized Eisenstein series $E_{a b}^{n, *}\left(\phi_{\Delta(\tau, b)}, s\right)$ is holomorphic at the point $s=0$.

To determine the location of possible poles of the normalized Eisenstein series $E_{a b}^{n, *}\left(\phi_{\Delta(\tau, b)}, s\right)$ for $b>1$, we consider the following four cases:

(1) $L(s, \tau, \rho)$ has a pole at $s=1$, and $L\left(\frac{1}{2}, \tau\right) \neq 0$ if $G_{n}$ is of Type (1);

(2) if $G_{n}$ is of Type (1), then $L(s, \tau, \rho)$ has a pole at $s=1$ and $L\left(\frac{1}{2}, \tau\right)=0$;

(3) if $G_{n}$ is of Type (1), then $L\left(s, \tau, \rho^{-}\right)$has a pole at $s=1$ and $L(s, \tau)$ has a pole at $s=1$ (this case occurs only if $a=1$ and $\tau$ is the trivial character of $\left.\mathrm{GL}_{1}(\mathbb{A})\right)$;

(4) $L\left(s, \tau, \rho^{-}\right)$has a pole at $s=1$, and $L(s, \tau)$ is holomorphic at $s=1$ if $G_{n}$ is of Type (1).

Note that in Type (1), $G_{n}=\mathrm{Sp}_{2 n}$, and in Type (2), $G_{n}=\mathrm{SO}_{2 n+1}$ or $\mathrm{SO}_{2 n}$. When $a=1$ and $\tau$ is a quadratic character of $\mathrm{GL}_{1}(\mathbb{A})$, [Kudla and Rallis 1990; 1994] treat the case when $G_{n}=\mathrm{Sp}_{2 n}$ or $\mathrm{SO}_{2 n}$.

Similarly, we define the sets of possible poles according to the four cases:

$$
X_{b, \tau}^{+}:= \begin{cases}\left\{\hat{0}, \ldots, \frac{b-2}{2}, \frac{b}{2}\right\}, & \text { in Case (1); } \\ \left\{\hat{0}, \ldots, \frac{b-4}{2}, \frac{b-2}{2}\right\}, & \text { in Case (2); } \\ \left\{\hat{0}, \ldots, \frac{b-1}{2}, \frac{b+1}{2}\right\}, & \text { in Case (3); } \\ \left\{\hat{0}, \ldots, \frac{b-3}{2}, \frac{b-1}{2}\right\}, & \text { in Case (4). }\end{cases}
$$

We also omit 0 because $E_{a b}^{n, *}\left(\phi_{\Delta(\tau, b)}, s\right)$ is holomorphic at $s=0$ (Proposition 5.1).

Now the same inductive argument proves Theorem 1.2 for the case of $m=0$ and $b>1$. We omit the details here.

Theorem 5.2 (case $m=0$ of Theorem 1.2). Let $G_{n}$ be a symplectic group or $F$-quasisplit orthogonal group. Assume that the irreducible unitary cuspidal automorphic representation $\tau$ of $\mathrm{GL}_{a}(\mathbb{A})$ is self-dual. Then the normalized Eisenstein series $E_{a b}^{n, *}\left(\phi_{\Delta(\tau, b)}, s\right)$ is holomorphic for $\operatorname{Re}(s) \geq 0$ except possibly at most simple poles at $s=s_{0} \in X_{b, \tau}^{+}$. 


\section{Residual representations and Arthur parameters}

In this section, we assume that $G_{n}$ is either symplectic or orthogonal, since we will use results from [Arthur 2013]. Based on Theorem 1.2, we will check the square-integrability for the residues at $s_{0} \in X_{b, \tau, \sigma}^{+}$of the normalized Eisenstein series $E_{a b}^{n, *}\left(\phi_{\Delta(\tau, b) \otimes \sigma}, s\right)$ (including the case of $\left.m=0\right)$ in Section 6A, and write down the Arthur parameters for those square-integrable residual representations if they are nonzero in Section 6B. Based on Sections 6A and 6B, we prove Proposition 4.4 using the Arthur classification [2013] of discrete spectrum. Finally, we investigate the conditions for the nonvanishing of those residual representations.

6A. Square-integrability. We recall that $P_{a^{b}, m}=M_{a^{b}, m} N_{a^{b}, m}$ is the standard parabolic subgroup of $G_{n}$ whose Levi subgroup is isomorphic to $\mathrm{GL}_{a}^{\times b} \times G_{m}$. Simply denote by $\Delta_{b}:=\Delta_{M_{a^{b}, m}}$ the set of restricted simple roots that can be described as follows.

Let $\left\{e_{i} \mid 1 \leq i \leq b\right\}$ be the natural set of coordinates on $\operatorname{Re} \mathfrak{a}_{M_{a^{b}, m}}^{*}$. If $G_{m}$ is not trivial, then

$$
\Delta_{b}=\left\{e_{1}-e_{2}, e_{2}-e_{3}, \ldots, e_{b-1}-e_{b}, e_{b}\right\} .
$$

If $G_{m}$ is trivial, then $\Delta_{b}=\Delta_{0}$, where $\Delta_{0}$ is the set of simple roots of $R\left(T_{0}, G_{b}\right)$.

Recall the notation in Section I.3 of [Mœglin and Waldspurger 1995]. Let $\phi$ be an automorphic function and let $\Pi_{0}(M, \phi)$ be the cuspidal support of $\phi$ along $P=M N$. The cuspidal exponent $\operatorname{Re}(\pi)$ for $\pi$ in $\Pi_{0}(M, \phi)$ is realized as a vector in terms of the basis $\left\{e_{i} \mid 1 \leq i \leq b\right\}$. Denote the cuspidal exponent of $\phi$ by

$$
e(\phi)=\left\{\operatorname{Re}(\pi) \mid \text { for all } \pi \in \Pi_{0}(M, \phi) \text { and for all } P=M N\right\} .
$$

Let $e\left(s_{0}, b, \tau, \sigma\right)$ be the set of cuspidal exponents of the residues of the normalized Eisenstein series $E_{a b}^{n, *}\left(\phi_{\Delta(\tau, b) \otimes \sigma}, s\right)$ at $s=s_{0}$ belonging to the set $X_{b, \tau, \sigma}^{+}$.

By the square-integrability criterion [Mœglin and Waldspurger 1995, Lemma I.4.11], the residues of the Eisenstein series are square-integrable if and only if each character of cuspidal support can be written in the form

$$
\sum_{\alpha \in \Delta_{M}} x_{\alpha} \alpha
$$

with coefficients $x_{\alpha} \in \mathbb{R}, x_{\alpha}<0$. Moreover, in our cases the criterion is equivalent to, for all $\sum_{i=1}^{b} c_{i} e_{i}$ in $e\left(s_{0}, b, \tau, \sigma\right)$,

$$
\sum_{i=1}^{j} c_{i}<0 \quad \text { for all } 1 \leq j \leq b .
$$

Theorem 6.1 (square-integrability). Let $s_{0} \in \mathbb{C}$ such that $\operatorname{Re}\left(s_{0}\right)$ is in $(0,(b+1) / 2]$. Assume that the normalized Eisenstein series $E_{a b}^{n, *}\left(\phi_{\Delta(\tau, b) \otimes \sigma}, s\right)$ has a simple pole 
at $s=s_{0}$. Then the residue of $E_{a b}^{n, *}\left(\phi_{\Delta(\tau, b) \otimes \sigma}, s\right)$ at $s_{0}$ is square-integrable except at $s_{0}=(b-1) / 2$ in Case (3).

Proof. The theorem is proved by induction on $b$. The key step in the proof is to determine the cuspidal exponents in $e\left(s_{0}, b, \tau, \sigma\right)$ by applying the induction formula (3-8), Lemma 2.1, and Lemma 2.2.

First, when $b=1$, by Section $4 \mathrm{~A}$, if the Eisenstein series has a pole at $s_{0}>0$, then the cuspidal exponent of the residue of the Eisenstein series is $-s_{0}$ and the residue is square-integrable. By Proposition 4.1, the Eisenstein series is holomorphic at $\operatorname{Re}(s) \geq 0$ except at $s=\frac{1}{2}$ or $s=1$. In these cases, the cuspidal exponent of the residues of the Eisenstein series is $-s_{0}=-\frac{1}{2}$ or -1 . Then $e\left(s_{0}, 1, \tau, \sigma\right)$ satisfies the condition (6-1) and the residues are square-integrable. Hence, the statement is true for $b=1$.

Next, we assume that the statement holds for $b-1$ and show that it is also true for $b$ by induction.

By the induction formula (3-8), we have to consider the cuspidal exponents of the two terms

$$
L\left(2 s+1, \tau, \rho^{(-)^{b+1}}\right) E_{a(b-1)}^{n-a, *}\left(\cdot, s+\frac{1}{2}\right)
$$

and

$$
L\left(2 s, \tau, \rho^{(-)^{b+1}}\right) E_{a(b-1)}^{n-a, *}\left(\cdot, s-\frac{1}{2}\right) .
$$

If $(b-1) / 2<\operatorname{Re}(s) \leq(b+1) / 2$, the first term $E_{a(b-1)}^{n-a, *}\left(\cdot, s+\frac{1}{2}\right)$ is holomorphic. Since $b \geq 2$ and $E_{a b}^{n, *}\left(\phi_{\Delta(\tau, b) \otimes \sigma}, s\right)$ has a pole at $s_{0}$, the second term $E_{a(b-1)}^{n-a, *}\left(\cdot, s-\frac{1}{2}\right)$ has a pole at $s_{0}$. By Lemma 2.2, the set $e\left(s_{0}, b, \tau, \sigma\right)$ of the cuspidal exponents equals

$$
\left\{\left(-s_{0}-\frac{b-1}{2}, c_{1}, \ldots, c_{b-1}\right) \mid\left(c_{1}, \ldots, c_{b-1}\right) \in e\left(s_{0}-\frac{1}{2}, b-1, \tau, \sigma\right)\right\} .
$$

By induction, $e\left(s_{0}-\frac{1}{2}, b-1, \tau, \sigma\right)$ satisfies the condition (6-1). It follows that $e\left(s_{0}, b, \tau, \sigma\right)$ also satisfies the condition (6-1). Hence the residue of the Eisenstein series $E_{a b}^{n, *}\left(\phi_{\Delta(\tau, b) \otimes \sigma}, s\right)$ at $s_{0}$ is square-integrable.

Next we consider the points at $0<\operatorname{Re}(s) \leq(b-1) / 2$. By the normalized induction formula (3-8), Lemma 2.1, and Lemma 2.2, the set of cuspidal exponents $e\left(s_{0}, b, \tau, \sigma\right)$ is a subset of the union

$$
\begin{aligned}
& \left\{\left(s_{0}-\frac{b-1}{2}, c_{1}, \ldots, c_{b-1}\right) \mid\left(c_{1}, \ldots, c_{b-1}\right) \in e\left(s_{0}+\frac{1}{2}, b-1, \tau, \sigma\right)\right\} \\
& \cup\left\{\left(-s_{0}-\frac{b-1}{2}, c_{1}, \ldots, c_{b-1}\right) \mid\left(c_{1}, \ldots, c_{b-1}\right) \in e\left(s_{0}-\frac{1}{2}, b-1, \tau, \sigma\right)\right\} .
\end{aligned}
$$

When $s_{0}=\frac{1}{2}$, the set $e\left(s_{0}-\frac{1}{2}, b-1, \tau, \sigma\right)$ needs some explanation. If $s_{0}=\frac{1}{2}$ and $E_{a(b-1)}^{n-a, *}\left(\phi_{\Delta(\tau, b-1) \otimes \sigma}, s-\frac{1}{2}\right)$ in the second term vanishes at $s=s_{0}$, then the second 
term is holomorphic at $s=\frac{1}{2}$. Hence we do not need to consider this set of cuspidal exponents when we only consider the square-integrability for nonzero residues. On the other hand, if $E_{a(b-1)}^{n-a, *}\left(\phi_{\Delta(\tau, b-1) \otimes \sigma}, s-\frac{1}{2}\right)$ in the second term does not vanish at $s_{0}$, by Section $1 \mathrm{~B}$, then the cuspidal exponent of $E_{a(b-1)}^{n-a, *}\left(\cdot, s-\frac{1}{2}\right)$ at $s_{0}$ is

$$
e\left(s_{0}-\frac{1}{2}, b-1, \tau, \sigma\right)=\left\{\left(\frac{2-b}{2}, \frac{4-b}{2}, \ldots, \frac{b-2}{2}\right)\right\} .
$$

When $s_{0}=(b-1) / 2$ in Case (3), the residue of the first term of the normalized induction formula is nonzero due to the nonvanishing of the residue of

$$
E_{a(b-1)}^{n-a, *}\left(\phi_{\Delta(\tau, b-1) \otimes \sigma}, s\right)
$$

at $s=b / 2$ in Theorem 6.2. Then $e\left(s_{0}, b, \tau, \sigma\right)$ contains the set

$$
\left\{\left(s_{0}-\frac{b-1}{2}, c_{1}, \ldots, c_{b-1}\right) \mid\left(c_{1}, \ldots, c_{b-1}\right) \in e\left(s_{0}+\frac{1}{2}, b-1, \tau, \sigma\right)\right\},
$$

which does not satisfy the condition (6-1), but satisfies $\sum_{i=1}^{j} c_{i} \leq 0$.

When $s_{0}=(b-1) / 2$, but not in Case (3), then the first term in the induction formula, $E_{a(b-1)}^{n-a, *}\left(\cdot, s+\frac{1}{2}\right)$, is holomorphic at $s=(b-1) / 2$. Thus, only the second term $E_{a(b-1)}^{n-a, *}\left(\cdot, s-\frac{1}{2}\right)$ has a possible pole at $s=(b-1) / 2$. Then $e\left(s_{0}, b, \tau, \sigma\right)$ equals

$$
\left\{\left(-s_{0}-\frac{b-1}{2}, c_{1}, \ldots, c_{b-1}\right) \mid\left(c_{1}, \ldots, c_{b-1}\right) \in e\left(s_{0}-\frac{1}{2}, b-1, \tau, \sigma\right)\right\},
$$

whose vectors satisfy the square-integrability criterion.

For $s_{0}<(b-1) / 2$, we have $s_{0}-\frac{1}{2}<(b-2) / 2$ and $s_{0}+\frac{1}{2}<b / 2$. Since $-s_{0}-(b-1) / 2<0$ and $s_{0}-(b-1) / 2<0$, by induction, each vector in the set $(6-2)$ satisfies the square-integrability criterion. This completes the proof.

6B. Arthur parameters. From Theorem 6.1, the residual representations of $G_{n}(\mathbb{A})$ generated by the residues of the (normalized) Eisenstein series $E_{a b}^{n, *}\left(\phi_{\Delta(\tau, b) \otimes \sigma}, s\right)$ at $s=s_{0}$ belonging to the set $X_{b, \tau, \sigma}^{+}$belong to the discrete spectrum of the space of automorphic forms on $G_{n}(\mathbb{A})$, except one case when $s_{0}=(b-1) / 2$ for Case (3). Denote the residual representation by $\mathscr{E} \Delta(\tau, b) \otimes \sigma, s_{0}$.

We will figure out the Arthur parameters for those square-integrable residual representations $\mathscr{E}_{\Delta}(\tau, b) \otimes \sigma, s_{0}$ if they are nonzero. Note that the nonvanishing conditions for those residual representations will be studied in the next subsection. We do this case by case for $s_{0} \in X_{b, \tau, \sigma}^{+}$.

We assume $\sigma$ is an irreducible cuspidal automorphic representation of $G_{m}(\mathbb{A})$ with tempered global Arthur parameter $\psi_{\sigma}$ [2013].

Case (1): In this case, when $m>0$, the irreducible unitary cuspidal automorphic representation $\tau$ of $\mathrm{GL}_{a}(\mathbb{A})$ has the property that $L(s, \tau, \rho)$ has a simple pole at 
$s=1$ and $L\left(\frac{1}{2}, \tau \times \sigma\right) \neq 0$, where $\rho$ is the symmetric square representation of $\mathrm{GL}_{a}(\mathbb{C})$ if $G_{n}=\mathrm{SO}_{2 n+1}$, and is the exterior square representation of $\mathrm{GL}_{a}(\mathbb{C})$ if $G_{n}$ is $\mathrm{Sp}_{2 n}$ or $\mathrm{SO}_{2 n}$.

We consider the residual representation $\mathscr{E}_{\Delta}(\tau, b) \otimes \sigma, s_{0}$ of $G_{n}(\mathbb{A})$ at $s_{0}=(b-2 j) / 2$ with $j=0,1, \ldots,[(b-1) / 2]$. According to [Arthur 2013], the global Arthur parameter $\psi$ attached to the residual representation $\mathscr{E}_{\Delta(\tau, b) \otimes \sigma, s_{0}}$ of $G_{n}(\mathbb{A})$ is

$$
\psi=\psi_{\Delta(\tau, b) \otimes \sigma,(b-2 j) / 2}=(\tau, 2(b-j)) \boxplus(\tau, 2 j) \boxplus \psi_{\sigma},
$$

with $j=0,1, \ldots,[(b-1) / 2]$. Note that when $G_{n}$ is $\mathrm{SO}_{2 n+1}, \tau$ is of orthogonal type; and when $G_{n}$ is $\mathrm{Sp}_{2 n}$ or $\mathrm{SO}_{2 n}, \tau$ is of symplectic type. Thus $\psi_{\Delta(\tau, b) \otimes \sigma,(b-2 j) / 2}$ is a global Arthur parameter for $G_{n}$. When $m=0$, we have

$$
\psi=\psi_{\Delta(\tau, b) \otimes \sigma,(b-2 j) / 2}= \begin{cases}(\tau, 2(b-j)) \boxplus(\tau, 2 j) & \text { if } G_{n} \neq \mathrm{Sp}_{2 n}, \\ (\tau, 2(b-j)) \boxplus(\tau, 2 j) \boxplus\left(1_{\mathrm{GL}_{1}(\mathbb{A})}, 1\right) & \text { if } G_{n}=\mathrm{Sp}_{2 n} .\end{cases}
$$

Case (2): This case is the same as Case (1), and the only difference is that $s_{0}=$ $(b-2 j) / 2$ with $j=1,2, \ldots,[(b-1) / 2]$. Hence when $m>0$, the global Arthur parameter $\psi$ attached to the residual representation $\mathscr{E}_{\Delta(\tau, b) \otimes \sigma, s_{0}}$ of $G_{n}(\mathbb{A})$ is

$$
\psi=\psi_{\Delta(\tau, b) \otimes \sigma,(b-2 j) / 2}=(\tau, 2(b-j)) \boxplus(\tau, 2 j) \boxplus \psi_{\sigma},
$$

with $j=1,2, \ldots,[(b-1) / 2]$; and when $m=0$, we have

$$
\psi=\psi_{\Delta(\tau, b) \otimes \sigma,(b-2 j) / 2}= \begin{cases}(\tau, 2(b-j)) \boxplus(\tau, 2 j) & \text { if } G_{n} \neq \mathrm{Sp}_{2 n}, \\ (\tau, 2(b-j)) \boxplus(\tau, 2 j) \boxplus\left(1_{\mathrm{GL}_{1}(\mathbb{A})}, 1\right) & \text { if } G_{n}=\mathrm{Sp}_{2 n} .\end{cases}
$$

Case (3): In this case, when $m>0$, the irreducible unitary cuspidal automorphic representation $\tau$ of $\mathrm{GL}_{a}(\mathbb{A})$ has the property that $L\left(s, \tau, \rho^{-}\right)$has a simple pole at $s=1$ and $L(s, \tau \times \sigma)$ also has a simple pole at $s=1$, where $\rho^{-}$is the exterior square representation of $\mathrm{GL}_{a}(\mathbb{C})$ if $G_{n}=\mathrm{SO}_{2 n+1}$, and is the symmetric square representation of $\mathrm{GL}_{a}(\mathbb{C})$ if $G_{n}$ is $\mathrm{Sp}_{2 n}$ or $\mathrm{SO}_{2 n}$. Following [Arthur 2013], the global tempered Arthur parameter for the irreducible cuspidal automorphic representation $\sigma$ is

$$
\psi_{\sigma}=(\tau, 1) \boxplus \psi^{\prime},
$$

where $\psi^{\prime}$ is a global Arthur parameter that is the complement of $(\tau, 1)$ in $\psi_{\sigma}$.

We consider the residual representation $\mathscr{E}_{\Delta(\tau, b) \otimes \sigma, s_{0}}$ of $G_{n}(\mathbb{A})$ at

$$
s_{0}=\frac{b+1-2 j}{2}
$$

with $j=0,1, \ldots,[b / 2]$. According to [Arthur 2013], the global Arthur parameter $\psi$ attached to the residual representation $\mathscr{E}_{\Delta(\tau, b) \otimes \sigma, s_{0}}$ of $G_{n}(\mathbb{A})$ is

$$
\psi=\psi_{\Delta(\tau, b) \otimes \sigma,(b+1-2 j) / 2}=(\tau, 2 b+1-2 j) \boxplus(\tau, 2 j-1) \boxplus \psi_{\sigma},
$$


with $j=2,3, \ldots,[b / 2]$. If $j=0$, we have

$$
\psi=\psi_{\Delta(\tau, b) \otimes \sigma,(b+1) / 2}=(\tau, 2 b+1) \boxplus \psi^{\prime} .
$$

Note that when $j=1$, the residual representation $\mathscr{E}_{\Delta(\tau, b) \otimes \sigma, s_{0}=(b-1) / 2}$ of $G_{n}(\mathbb{A})$ is not square-integrable (Theorem 6.1). Note that when $G_{n}$ is $\mathrm{SO}_{2 n+1}, \tau$ is of symplectic type; and when $G_{n}$ is $\mathrm{Sp}_{2 n}$ or $\mathrm{SO}_{2 n}, \tau$ is of orthogonal type. Hence $\psi_{\Delta(\tau, b) \otimes \sigma,(b-2 j) / 2}$ with $j=0$ or $j=2,3, \ldots,[b / 2]$ is a global Arthur parameter for $G_{n}$.

When $m=0$, this case only occurs if $a=1$ and $\tau$ is the trivial representation of $\mathrm{GL}_{1}(\mathbb{A})$. If $j=2,3, \ldots,[b / 2]$, we have

$$
\psi=\psi_{\Delta(\tau, b) \otimes \sigma,(b+1-2 j) / 2}=(\tau, 2 b+1-2 j) \boxplus(\tau, 2 j-1) \boxplus\left(1_{\mathrm{GL}_{1}(\mathbb{A})}, 1\right) .
$$

If $j=0$, according to the definition of the four cases, $G_{n}$ must be $\mathrm{Sp}_{2 n}$ and $\psi=\psi_{\Delta(\tau, b) \otimes \sigma,(n+1) / 2}=(\tau, 2 n+1)$.

Case (4): This case is similar to Case (3). The only difference is that

$$
s_{0}=\frac{b+1-2 j}{2}
$$

with $j=1,2, \ldots,[b / 2]$. Hence when $m>0$, the global Arthur parameter $\psi$ attached to the residual representation $\mathscr{E}_{\Delta(\tau, b) \otimes \sigma, s_{0}}$ of $G_{n}(\mathbb{A})$ is

$$
\psi=\psi_{\Delta(\tau, b) \otimes \sigma,(b+1-2 j) / 2}=(\tau, 2 b+1-2 j) \boxplus(\tau, 2 j-1) \boxplus \psi_{\sigma},
$$

with $j=1,2,3, \ldots,[b / 2]$, and when $m=0$, we have

$$
\begin{aligned}
\psi & =\psi_{\Delta(\tau, b) \otimes \sigma,(b+1-2 j) / 2} \\
& = \begin{cases}(\tau, 2 b+1-2 j) \boxplus(\tau, 2 j-1) & \text { if } G_{n} \neq \mathrm{Sp}_{2 n}, \\
(\tau, 2 b+1-2 j) \boxplus(\tau, 2 j-1) \boxplus\left(1_{\mathrm{GL}_{1}(\mathbb{A})}, 1\right) & \text { if } G_{n}=\mathrm{Sp}_{2 n} .\end{cases}
\end{aligned}
$$

Note that the residual representation $\mathscr{E}_{\Delta(\tau, b) \otimes \sigma,(b-1) / 2}$ of $G_{n}(\mathbb{A})(j=1)$ in this case belongs to the discrete spectrum of $G_{n}(\mathbb{A})$.

6C. Proof of Proposition 4.4. Proposition 4.4 follows from the discussion on square-integrability in Section 6A and the discussion on the global Arthur parameter in Section 6B. In fact, if there is an $s_{0}$ such that $0<\operatorname{Re}\left(s_{0}\right)<\frac{1}{2}$, such that the normalized Eisenstein series $E_{a b}^{n, *}\left(\phi_{\Delta(\tau, b) \otimes \sigma}, s\right)$ has a pole at $s=s_{0}$, then by Theorem 6.1, the residue at $s=s_{0}$ must be square-integrable, and hence the residual representation contributes to the discrete spectrum. On the other hand, by the Arthur classification [2013] of the discrete spectrum, there is no global Arthur parameter for $G_{n}$ that parametrizes such a residual representation. Hence the normalized Eisenstein series $E_{a b}^{n, *}\left(\phi_{\Delta(\tau, b) \otimes \sigma}, s\right)$ must be holomorphic at $0<\operatorname{Re}(s)<\frac{1}{2}$. This proves Proposition 4.4. 
6D. Nonvanishing conditions. When $b=1$, the nonvanishing of the residues of the normalized Eisenstein series has been discussed in Proposition 4.1. In the following, we assume that $b>1$.

For $s=s_{0} \in X_{b, \tau, \sigma}^{+}$, the normalized Eisenstein series $E_{a b}^{n, *}\left(\phi_{\Delta(\tau, b) \otimes \sigma}, s\right)$ has a pole at $s=s_{0}$ if one of its constant terms has a pole at $s=s_{0}$. The normalized induction formula (3-8) says

$$
\begin{aligned}
& E_{a b, P_{a}}^{n, *}\left(\phi_{\Delta \otimes \sigma}, s\right)\left(\left(I_{a}, h\right)\right) \\
& =L\left(2 s+1, \tau, \rho^{\left.(-)^{b+1}\right)} E_{a(b-1)}^{n-a, *}\left(\lambda_{-1 / 2} i_{n-a}^{*} \phi_{Q}, s+\frac{1}{2}\right)(h)\right. \\
& \quad+\frac{L\left(2 s, \tau, \rho^{(-)^{b+1}}\right)}{\varepsilon_{b}^{\prime}(s)} E_{a(b-1)}^{n-a, *}\left(\lambda_{1 / 2}\left(i_{n-a}^{*} \circ N(\omega, \cdot)\right) \tilde{\phi}, s-\frac{1}{2}\right)(h) .
\end{aligned}
$$

Hence $s_{0}$ has the property that $s_{0} \in\left(X_{b-1, \tau, \sigma}^{+}+\frac{1}{2}\right) \cup\left(X_{b-1, \tau, \sigma}^{+}-\frac{1}{2}\right)$ and $s_{0}>0$.

By the discussion of the global Arthur parameters in Section $6 \mathrm{~B}$, if both

$$
E_{a(b-1)}^{n-a, *}\left(\lambda_{-1 / 2} i_{n-a}^{*} \phi_{Q}, s+\frac{1}{2}\right)(h)
$$

and

$$
E_{a(b-1)}^{n-a, *}\left(\lambda_{1 / 2}\left(i_{n-a}^{*} \circ N(\omega, \cdot)\right) \tilde{\phi}, s-\frac{1}{2}\right)(h)
$$

are nonzero, they cannot be proportional to each other since they have different Langlands parameters. Hence the problem reduces to verifying the nonvanishing of either of the two terms. Note that from the definition, both $\lambda_{-1 / 2} i_{n-a}^{*} \phi_{Q}$ and $\lambda_{1 / 2}\left(i_{n-a}^{*} \circ N(\omega, \cdot)\right) \tilde{\phi}$ give general sections in the corresponding space, respectively. Therefore, the existence of the poles of the normalized Eisenstein series $E_{a b}^{n, *}\left(\phi_{\Delta(\tau, b) \otimes \sigma}, s\right)$ at $s \in X_{b, \tau, \sigma}^{+}$follows from the existence of the poles of the normalized Eisenstein series $E_{a(b-1)}^{n, *}\left(\phi_{\Delta(\tau, b-1) \otimes \sigma}, s\right)$ at $s \in X_{b-1, \tau, \sigma}^{+}$.

By repeating the argument, this reduces to the case of $b$ being as small as possible. The discussion will be given for each of the four cases.

Case (1): In this case, the irreducible unitary cuspidal automorphic representation $\tau$ of $\mathrm{GL}_{a}(\mathbb{A})$ has the property that $L(s, \tau, \rho)$ has a simple pole at $s=1$ and

$$
L\left(\frac{1}{2}, \tau \times \sigma\right) \neq 0,
$$

where $\rho$ is the symmetric square representation of $\mathrm{GL}_{a}(\mathbb{C})$ if $G_{n}=\mathrm{SO}_{2 n+1}$, and the exterior square representation of $\mathrm{GL}_{a}(\mathbb{C})$ if $G_{n}$ is $\mathrm{Sp}_{2 n}$ or $\mathrm{SO}_{2 n}$. In this case, the smallest possible value of $b$ is $b=1$. The existence of the pole at the only value $s=\frac{1}{2}$ is treated in the first case in Proposition 4.1.

Case (2): In this case, the irreducible unitary cuspidal automorphic representation $\tau$ of $\mathrm{GL}_{a}(\mathbb{A})$ has the property that $L(s, \tau, \rho)$ has a simple pole at $s=1$ and

$$
L\left(\frac{1}{2}, \tau \times \sigma\right)=0,
$$


where $\rho$ is the symmetric square representation of $\mathrm{GL}_{a}(\mathbb{C})$ if $G_{n}=\mathrm{SO}_{2 n+1}$, and the exterior square representation of $\mathrm{GL}_{a}(\mathbb{C})$ if $G_{n}$ is $\mathrm{Sp}_{2 n}$ or $\mathrm{SO}_{2 n}$. In this case, the smallest possible value of $b$ is $b=3$, which leads us to consider the existence of the pole at $s=\frac{1}{2}$. The normalized induction formula is

$$
\begin{aligned}
& E_{3 a, P_{a}}^{n, *}\left(\phi_{\Delta \otimes \sigma}, s\right)\left(\left(I_{a}, h\right)\right) \\
& =L(2 s+1, \tau, \rho) E_{2 a}^{n-a, *}\left(\lambda_{-1 / 2} i_{n-a}^{*} \phi_{Q}, s+\frac{1}{2}\right)(h) \\
& \quad+\frac{L(2 s, \tau, \rho)}{\varepsilon_{3}^{\prime}(s)} E_{2 a}^{n-a, *}\left(\lambda_{1 / 2}\left(i_{n-a}^{*} \circ N(\omega, \cdot)\right) \tilde{\phi}, s-\frac{1}{2}\right)(h) .
\end{aligned}
$$

It follows from Theorem 1.2 that the first term

$$
L(2 s+1, \tau, \rho) E_{2 a}^{n-a, *}\left(\lambda_{-1 / 2} i_{n-a}^{*} \phi_{Q}, s+\frac{1}{2}\right)(h)
$$

is holomorphic at $s=\frac{1}{2}$, since $L(2 s+1, \tau, \rho)$ is holomorphic at $s=\frac{1}{2}$ and $s+\frac{1}{2}=1$ does not belong to the empty set $X_{\tau, 2, \sigma}^{+}$in the case of the normalized Eisenstein series $E_{2 a}^{n-a, *}\left(\lambda_{-1 / 2} i_{n-a}^{*} \phi_{Q}, s+\frac{1}{2}\right)(h)$.

The second term

$$
\frac{L(2 s, \tau, \rho)}{\varepsilon_{3}^{\prime}(s)} E_{2 a}^{n-a, *}\left(\lambda_{1 / 2}\left(i_{n-a}^{*} \circ N(\omega, \cdot)\right) \tilde{\phi}, s-\frac{1}{2}\right)(h)
$$

has a simple pole at $s=\frac{1}{2}$ if and only if the normalized Eisenstein series

$$
E_{2 a}^{n-a, *}\left(\phi_{\Delta(\tau, 2) \otimes \sigma}, s\right)
$$

is not identically zero at $s=0$.

Case (3): In this case, the irreducible unitary cuspidal automorphic representation $\tau$ of $\mathrm{GL}_{a}(\mathbb{A})$ has the property that $L\left(s, \tau, \rho^{-}\right)$has a simple pole at $s=1$ and that $L(s, \tau \times \sigma)$ also has a simple pole at $s=1$, where $\rho^{-}$is the exterior square representation of $\mathrm{GL}_{a}(\mathbb{C})$ if $G_{n}=\mathrm{SO}_{2 n+1}$, and the symmetric square representation of $\mathrm{GL}_{a}(\mathbb{C})$ if $G_{n}$ is $\mathrm{Sp}_{2 n}$ or $\mathrm{SO}_{2 n}$. In this case, the smallest possible value of $b$ is $b=1$. The existence of the pole at the only value $s=1$ is treated in the second case in Proposition 4.1.

Case (4): In this case, the irreducible unitary cuspidal automorphic representation $\tau$ of $\mathrm{GL}_{a}(\mathbb{A})$ has the property that $L\left(s, \tau, \rho^{-}\right)$has a simple pole at $s=1$ and $L(s, \tau \times \sigma)$ is holomorphic at $s=1$, where $\rho^{-}$is the exterior square representation of $\mathrm{GL}_{a}(\mathbb{C})$ if $G_{n}=\mathrm{SO}_{2 n+1}$, and the symmetric square representation of $\mathrm{GL}_{a}(\mathbb{C})$ if $G_{n}$ is $\mathrm{Sp}_{2 n}$ or $\mathrm{SO}_{2 n}$. In this case, the smallest possible value of $b$ is $b=2$, which leads us to consider the existence of the pole at $s=\frac{1}{2}$. The normalized induction 
formula is

$$
\begin{aligned}
E_{2 a, P_{a}}^{n, *}\left(\phi_{\Delta \otimes \sigma}, s\right)\left(\left(I_{a}, h\right)\right) & \\
=L(2 s+ & \left.1, \tau, \rho^{-}\right) E_{a}^{n-a, *}\left(\lambda_{-1 / 2} i_{n-a}^{*} \phi_{Q}, s+\frac{1}{2}\right)(h) \\
& +\frac{L\left(2 s, \tau, \rho^{-}\right)}{\varepsilon_{2}^{\prime}(s)} E_{a}^{n-a, *}\left(\lambda_{1 / 2}\left(i_{n-a}^{*} \circ N(\omega, \cdot)\right) \tilde{\phi}, s-\frac{1}{2}\right)(h) .
\end{aligned}
$$

It follows from Theorem 1.2 that the first term

$$
L\left(2 s+1, \tau, \rho^{-}\right) E_{a}^{n-a, *}\left(\lambda_{-1 / 2} i_{n-a}^{*} \phi_{Q}, s+\frac{1}{2}\right)
$$

is holomorphic at $s=\frac{1}{2}$ with the same argument as in Case (2). The second term

$$
\frac{L\left(2 s, \tau, \rho^{-}\right)}{\varepsilon_{2}^{\prime}(s)} E_{a}^{n-a, *}\left(\lambda_{1 / 2}\left(i_{n-a}^{*} \circ N(\omega, \cdot)\right) \tilde{\phi}, s-\frac{1}{2}\right)
$$

has a simple pole at $s=\frac{1}{2}$ if and only if the normalized Eisenstein series

$$
E_{a}^{n-a, *}\left(\phi_{\tau \otimes \sigma}, s\right)
$$

does not vanish identically at $s=0$.

The above discussion leads to the following theorem.

Theorem 6.2. With the notation above, the following hold.

(1) Assume that $L\left(\frac{1}{2}, \tau \times \sigma\right) \neq 0$ and $L(s, \tau, \rho)$ has a simple pole at $s=1$. The normalized Eisenstein series

$$
E_{a b}^{n, *}\left(\phi_{\Delta(\tau, b) \otimes \sigma}, s\right)
$$

has a simple pole at each $s \in X_{b, \tau, \sigma}^{+}$, which is defined in Case (1).

(2) Assume that $L\left(\frac{1}{2}, \tau \times \sigma\right)=0$ and $L(s, \tau, \rho)$ has a simple pole at $s=1$. The normalized Eisenstein series

$$
E_{a b}^{n, *}\left(\phi_{\Delta(\tau, b) \otimes \sigma}, s\right)
$$

has a simple pole at each $s \in X_{b, \tau, \sigma}^{+}$, which is defined in Case (2), if and only if the normalized Eisenstein series

$$
E_{2 a}^{n-a, *}\left(\phi_{\Delta(\tau, 2) \otimes \sigma}, s\right)
$$

is not identically zero at $s=0$.

(3) Assume that $L\left(s, \tau, \rho^{-}\right)$and $L(s, \tau \times \sigma)$ have a simple pole at $s=1$. The normalized Eisenstein series

$$
E_{a b}^{n, *}\left(\phi_{\Delta(\tau, b) \otimes \sigma}, s\right)
$$

has a simple pole at each $s \in X_{b, \tau, \sigma}^{+}$, which is defined in Case (3). 
(4) Assume that $L\left(s, \tau, \rho^{-}\right)$has a simple pole at $s=1$ and $L(s, \tau \times \sigma)$ is holomorphic at $s=1$. The normalized Eisenstein series

$$
E_{a b}^{n, *}\left(\phi_{\Delta(\tau, b) \otimes \sigma}, s\right)
$$

has a simple pole at each $s \in X_{b, \tau, \sigma}^{+}$, which is defined in Case (4), if and only if the normalized Eisenstein series

$$
E_{a}^{n-a, *}\left(\phi_{\tau \otimes \sigma}, s\right)
$$

does not vanish identically at $s=0$.

Remark 6.3. The nonvanishing results may also apply to the case of $m=0$ accordingly, but we omit the discussion here. Finally, it is natural to expect that the results discussed in this section hold for quasisplit unitary groups.

\section{Acknowledgements}

We would like to thank the referee for his/her careful reading of the previous version of this paper and very helpful comments and suggestions. We would also like to thank Erez Lapid for helpful conversations and communications on certain technical issues of this paper.

\section{References}

[Arthur 2013] J. Arthur, The endoscopic classification of representations: orthogonal and symplectic groups, Colloquium Publ., Amer. Math. Soc., Providence, 2013. To appear; preprint available at http://www.claymath.org/cw/arthur/pdf/Book.pdf.

[Brenner 2009] E. Brenner, "Analytic properties of residual Eisenstein series, I", J. Ramanujan Math. Soc. 24:4 (2009), 359-413. MR 2011a:11102 Zbl 1207.11060

[Cogdell and Piatetski-Shapiro 2004] J. W. Cogdell and I. I. Piatetski-Shapiro, "Remarks on RankinSelberg convolutions", pp. 255-278 in Contributions to automorphic forms, geometry, and number theory (Baltimore, MD, 2002), edited by H. Hida et al., Johns Hopkins University Press, Baltimore, MD, 2004. MR 2005d:11075 Zbl 1080.11038

[Cogdell et al. 2004] J. W. Cogdell, H. H. Kim, I. I. Piatetski-Shapiro, and F. Shahidi, "Functoriality for the classical groups", Publ. Math. Inst. Hautes Études Sci. 99 (2004), 163-233. MR 2006a:22010 Zbl 1090.22010

[Cogdell et al. 2011] J. W. Cogdell, I. I. Piatetski-Shapiro, and F. Shahidi, "Functoriality for the quasisplit classical groups", pp. 117-140 in On certain L-functions (West Lafayette, IN, 2007), edited by J. Arthur et al., Clay Math. Proc. 13, Amer. Math. Soc., Providence, RI, 2011. MR 2012f:22036 Zbl 1235.22025

[Ginzburg 2008] D. Ginzburg, "Endoscopic lifting in classical groups and poles of tensor $L$-functions", Duke Math. J. 141:3 (2008), 447-503. MR 2009c:22020 Zbl 1195.11068

[Ginzburg et al. 2011] D. Ginzburg, S. Rallis, and D. Soudry, The descent map from automorphic representations of GL(n) to classical groups, World Scientific, Singapore, 2011. MR 2012g:22020 Zbl 1233.11056 
[Ginzburg et al. 2012] D. Ginzburg, D. Jiang, and D. Soudry, "On correspondences between certain automorphic forms on $\mathrm{Sp}_{4 n}(\mathbb{A})$ and $\widetilde{\mathrm{Sp}}_{2 n}(\mathbb{A})$ )", Israel J. Math. 192:2 (2012), 951-1008. MR 3009748 Zbl pre06135123

[Grbac 2011] N. Grbac, "On the residual spectrum of split classical groups supported in the Siegel maximal parabolic subgroup", Monatsh. Math. 163:3 (2011), 301-314. MR 2805875 Zbl 05913913

[Jiang 1998] D. Jiang, "The first term identities for Eisenstein series”, J. Number Theory 70:1 (1998), 67-98. MR 99h:11052 Zbl 0913.11021

[Jiang 2011] D. Jiang, "Construction of endoscopy transfers for classical groups", in Automorphic forms: New directions, Oberwolfach Reports, EMS 8:1, March 2011. Available at http://www.math. umn.edu/ dhjiang/Papers/oberwolfach11.pdf.

[Jiang 2012] D. Jiang, "Automorphic integral transforms for classical groups I: endoscopy correspondences", preprint, 2012. To appear in Proceedings of the Conference on Automorphic Forms and Related Geometry: Assessing the legacy of I. I. Piatetski-Shapiro, edited by J. Cogdell et al. arXiv 1212.6525

[Jiang and Liu 2012] D. Jiang and B. Liu, "On Fourier coefficients of automorphic forms of GL(n)", Int. Math. Res. Not. 2012 (2012), article ID rns153. doi: 10.1093/imrn/rns153.

[Keys and Shahidi 1988] C. D. Keys and F. Shahidi, "Artin $L$-functions and normalization of intertwining operators”, Ann. Sci. École Norm. Sup. (4) 21:1 (1988), 67-89. MR 89k:22034 Zbl 0654.10030

[Kim and Krishnamurthy 2004] H. H. Kim and M. Krishnamurthy, "Base change lift for odd unitary groups", pp. 116-125 in Functional analysis VIII (Dubrovnik, 2003), edited by D. Bakić et al., Various Publ. Ser. (Aarhus) 47, Aarhus Univ., Aarhus, 2004. MR 2006a:11154 Zbl 1146.11313

[Kim and Krishnamurthy 2005] H. H. Kim and M. Krishnamurthy, "Stable base change lift from unitary groups to GL $n$ ”, Int. Math. Res. Pap. 2005:1 (2005), 1-52. MR 2006d:22028 Zbl 1146.11314

[Kudla and Rallis 1990] S. S. Kudla and S. Rallis, "Poles of Eisenstein series and $L$-functions", pp. 81-110 in Festschrift in honor of I. I. Piatetski-Shapiro on the occasion of his sixtieth birthday, II (Tel Aviv, 1989), edited by S. Gelbart et al., Israel Math. Conf. Proc. 3, Weizmann, Jerusalem, 1990. MR 94e:11054 Zbl 0712.11029

[Kudla and Rallis 1994] S. S. Kudla and S. Rallis, "A regularized Siegel-Weil formula: the first term identity”, Ann. of Math. (2) 140:1 (1994), 1-80. MR 95f:11036 Zbl 0818.11024

[Langlands 1976] R. P. Langlands, On the functional equations satisfied by Eisenstein series, Lecture Notes in Mathematics 544, Springer, Berlin, 1976. MR 58 \#28319 Zbl 0332.10018

[Lapid et al. 2004] E. Lapid, G. Muić, and M. Tadić, “On the generic unitary dual of quasisplit classical groups", Int. Math. Res. Not. 2004:26 (2004), 1335-1354. MR 2005b:22021 Zbl 1079.22015

[Luo et al. 1999] W. Luo, Z. Rudnick, and P. Sarnak, "On the generalized Ramanujan conjecture for GL(n)", pp. 301-310 in Automorphic forms, automorphic representations, and arithmetic (Fort Worth, TX, 1996), edited by R. S. Doran et al., Proc. Sympos. Pure Math. 66, Part 2, Amer. Math. Soc., Providence, RI, 1999. MR 2000e:11072 Zbl 0965.11023

[Mœglin 2008] C. Mœglin, "Formes automorphes de carré intégrable non cuspidales", Manuscripta Math. 127:4 (2008), 411-467. MR 2010i:11071 Zbl 05520091

[Mœglin 2010] C. Mœglin, "Holomorphie des opérateurs d'entrelacement normalisés à l'aide des paramètres d'Arthur", Canad. J. Math. 62:6 (2010), 1340-1386. MR 2012c:22024 Zbl 1204.22012

[Mœglin and Waldspurger 1989] C. Mœglin and J.-L. Waldspurger, "Le spectre résiduel de GL(n)", Ann. Sci. École Norm. Sup. (4) 22:4 (1989), 605-674. MR 91b:22028 Zbl 0696.10023 
[Mœglin and Waldspurger 1995] C. Mœglin and J.-L. Waldspurger, Spectral decomposition and Eisenstein series, Cambridge Tracts in Mathematics 113, Cambridge University Press, Cambridge, 1995. MR 97d:11083 Zbl 0846.11032

[Mok 2012] C. P. Mok, "Endoscopic classification of representations of quasi-split unitary groups I", preprint, 2012. arXiv 1206.0882

[Shahidi 2010] F. Shahidi, Eisenstein series and automorphic L-functions, Amer. Math. Soc. Colloq. Publ. 58, Amer. Math. Soc., Providence, RI, 2010. MR 2012d:11119 Zbl 1215.11054

Received January 27, 2012. Revised July 20, 2012.

DIHUA JIANG

SCHOOL OF MATHEMATICS

UNIVERSITY OF MINNESOTA

MINNEAPOLIS, MN 55455

UNITED STATES

dhjiang@math.umn.edu

BAIYING LIU

SCHOOL OF MATHEMATICS

UNIVERSITY OF MINNESOTA

MINNEAPOLIS, MN 55455

UNITED STATES

liuxx969@umn.edu

LEI ZHANG

DEPARTMENT OF MATHEMATICS

Boston College

Carney Hall, 140 Commonwealth Ave

Chestnut Hill, MA 02467

UNITED STATES

lei.zhang.2@bc.edu 


\title{
PACIFIC JOURNAL OF MATHEMATICS
}

\author{
msp.org/pjm
}

Founded in 1951 by E. F. Beckenbach (1906-1982) and F. Wolf (1904-1989)

\section{EDITORS}

V. S. Varadarajan (Managing Editor)

Department of Mathematics

University of California

Los Angeles, CA 90095-1555

pacific@math.ucla.edu

Paul Balmer

Department of Mathematics

University of California

Los Angeles, CA 90095-1555

balmer@math.ucla.edu

Daryl Cooper

Department of Mathematics

University of California

Santa Barbara, CA 93106-3080 cooper@math.ucsb.edu

Jiang-Hua $\mathrm{Lu}$

Department of Mathematics

The University of Hong Kong

Pokfulam Rd., Hong Kong jhlu@maths.hku.hk
Don Blasius

Department of Mathematics University of California

Los Angeles, CA 90095-1555

blasius@math.ucla.edu

Robert Finn

Department of Mathematics Stanford University

Stanford, CA 94305-2125

finn@math.stanford.edu

Sorin Popa

Department of Mathematics

University of California

Los Angeles, CA 90095-1555

popa@math.ucla.edu

Paul Yang

Department of Mathematics

Princeton University

Princeton NJ 08544-1000

yang@math.princeton.edu

\section{PRODUCTION}

Silvio Levy, Scientific Editor, production@msp.org

\section{SUPPORTING INSTITUTIONS}

ACADEMIA SINICA, TAIPEI

CALIFORNIA INST. OF TECHNOLOGY

INST. DE MATEMÁTICA PURA E APLICADA

KEIO UNIVERSITY

MATH. SCIENCES RESEARCH INSTITUTE

NEW MEXICO STATE UNIV.

OREGON STATE UNIV.

\author{
STANFORD UNIVERSITY \\ UNIV. OF BRITISH COLUMBIA \\ UNIV. OF CALIFORNIA, BERKELEY \\ UNIV. OF CALIFORNIA, DAVIS \\ UNIV. OF CALIFORNIA, LOS ANGELES \\ UNIV. OF CALIFORNIA, RIVERSIDE \\ UNIV. OF CALIFORNIA, SAN DIEGO \\ UNIV. OF CALIF., SANTA BARBARA
}

\author{
Vyjayanthi Chari \\ Department of Mathematics \\ University of California \\ Riverside, CA 92521-0135 \\ chari@math.ucr.edu \\ Kefeng Liu \\ Department of Mathematics \\ University of California \\ Los Angeles, CA 90095-1555 \\ liu@math.ucla.edu \\ Jie Qing \\ Department of Mathematics \\ University of California \\ Santa Cruz, CA 95064 \\ qing@cats.ucsc.edu
}

These supporting institutions contribute to the cost of publication of this Journal, but they are not owners or publishers and have no responsibility for its contents or policies.

See inside back cover or msp.org/pjm for submission instructions.

The subscription price for 2013 is US \$400/year for the electronic version, and \$485/year for print and electronic.

Subscriptions, requests for back issues and changes of subscribers address should be sent to Pacific Journal of Mathematics, P.O. Box 4163, Berkeley, CA 94704-0163, U.S.A. The Pacific Journal of Mathematics is indexed by Mathematical Reviews, Zentralblatt MATH, PASCAL CNRS Index, Referativnyi Zhurnal, Current Mathematical Publications and the Science Citation Index.

The Pacific Journal of Mathematics (ISSN 0030-8730) at the University of California, c/o Department of Mathematics, 798 Evans Hall \#3840, Berkeley, CA 94720-3840, is published monthly except July and August. Periodical rate postage paid at Berkeley, CA 94704, and additional mailing offices. POSTMASTER: send address changes to Pacific Journal of Mathematics, P.O. Box 4163, Berkeley, CA 94704-0163.

PJM peer review and production are managed by EditFLOW ${ }^{\circledR}$ from Mathematical Sciences Publishers.

PUBLISHED BY

mathematical sciences publishers

nonprofit scientific publishing

http://msp.org/

(C) 2013 Mathematical Sciences Publishers 


\section{PACIFIC JOURNAL OF MATHEMATICS}

Volume $264 \quad$ No. $1 \quad$ July 2013

On the center of fusion categories

ALAIN BRUGUIÈRES and ALEXIS VIRELIZIER

Connected quandles associated with pointed abelian groups

W. EdWin Clark, MoHamed ElHamdadi, Xiang-DONG HoU,

MASAHICO SAITO and TIMOTHY YEATMAN

Entropy and lowest eigenvalue on evolving manifolds

HongXin GuO, ROBERT PHILIPOWSKI and ANTON THALMAIER

Poles of certain residual Eisenstein series of classical groups

DIHUA JIANG, BAIYING LIU and LEI ZHANG

Harmonic maps on domains with piecewise Lipschitz continuous metrics 125

HAIGANG LI and CHANGYOU WANG

$q$-hypergeometric double sums as mock theta functions

JEREMY LOVEJOY and ROBERT OSBURN

Monic representations and Gorenstein-projective modules

XIU-HuA LUO and PU ZHANG

Helicoidal flat surfaces in hyperbolic 3-space

Antonio Martínez, JoÃo Paulo dos Santos and Keti

TENENBLAT

On a Galois connection between the subfield lattice and the multiplicative subgroup lattice

JOHN K. MCVEY

Some characterizations of Campanato spaces via commutators on Morrey 221 spaces

Shaoguang Shi and Shanzhen Lu

The Siegel-Weil formula for unitary groups 